\title{
Global Attractor for 1D Dirac Field Coupled to Nonlinear Oscillator
}

\author{
Elena Kopylova $^{1,2}$, Alexander Komech ${ }^{1,2,3}$ \\ 1 Faculty of Mathematics of Vienna University, Vienna, Austria. E-mail: elena.kopylova@univie.ac.at. \\ 2 IITP RAS, Moscow, Russia \\ 3 Mechanics-Mathematics Department of Moscow State University, Moscow, Russia
}

Received: 20 November 2018 / Accepted: 24 March 2019

Published online: 8 May 2019 - (C) The Author(s) 2019

\begin{abstract}
Global attraction to solitary waves is proved for a model $\mathbf{U}(1)$-invariant nonlinear 1D Dirac equation coupled to a nonlinear oscillator: each finite energy solution converges as $t \rightarrow \pm \infty$ to a set of all "nonlinear eigenfunctions" of the form $\psi_{1}(x) e^{-i \omega_{1} t}+\psi_{2}(x) e^{-i \omega_{2} t}$. The global attraction is caused by nonlinear energy transfer from lower harmonics to continuous spectrum and subsequent dispersive radiation. We justify this mechanism by a strategy based on inflation of spectrum by the nonlinearity. We show that any omega-limit trajectory has the time-spectrum in the spectral gap $[-m, m]$ and satisfies the original equation. Then the application of the Titchmarsh convolution theorem reduces the spectrum of the omega-limit trajectory to two harmonics $\omega_{j} \in[-m, m], j=1,2$.
\end{abstract}

\section{Introduction}

The main goal of our paper is global attraction to solitary manifold for 1D Dirac equation with point coupling to an U(1)-invariant nonlinear oscillator. This goal is inspired by fundamental mathematical problem of an interaction between fields and point particles. Point interaction models were first considered since 1933 in the papers of Wigner, Bethe and Peierls, Fermi and others (see [2] for a detailed survey) and of Dirac [9]. Rigorous mathematical results were obtained since 1960 by Zeldovich, Berezin, Faddev, Cornish, Yafaev, Zeidler and others $[3,7,11,33,35]$, and since 2000 by Noja, Posilicano, Yafaev and others $[1,34]$.

In the case of the Maxwell field its coupling to a point particle is not well defined because the Hamilton functional is not bounded from below. This problem was resolved by Abraham by introduction of "extended electron" [31]. In the case of the Dirac equation the Hamilton functional also is not bounded from below even for the extended particle.

Elena Kopylova: Research supported by the Austrian Science Fund (FWF) under Grant No. P27492-N25 and RFBR Grant 18-01-00524.

Alexander Komech: Research supported by the Austrian Science Fund (FWF) under Grant No. P28152-N35. 
So one need to find an appropriate type of point interaction to the 1D Dirac equation which guarantees a priori estimates sufficient for the global attraction. We have found a novel model of such coupling which provides the Hamilton structure and needed a priori estimates. Namely, we consider the following Dirac equation

$$
i \dot{\psi}(x, t)=D_{m} \psi(x, t)-D_{m}^{-1} \delta(x) F(\psi(0, t)), \quad x \in \mathbb{R} .
$$

Here $D_{m}$ is the Dirac operator $D_{m}:=\alpha \partial_{x}+m \beta$, where $m>0$, and

$$
\alpha=\left(\begin{array}{cc}
0 & 1 \\
-1 & 0
\end{array}\right), \quad \beta=\left(\begin{array}{cc}
1 & 0 \\
0 & -1
\end{array}\right),
$$

$\psi(x, t)=\left(\psi_{1}(x, t), \psi_{2}(x, t)\right)$ is a continuous $\mathbb{C}^{2}$-valued wave function, and $F(\zeta)=$ $\left(F_{1}\left(\zeta_{1}\right), F_{2}\left(\zeta_{2}\right)\right), \zeta=\left(\zeta_{1}, \zeta_{2}\right) \in \mathbb{C}^{2}$, is a nonlinear vector function. The dots stand for the derivatives in $t$. All derivatives and Eq. (1.1) are understood in the sense of distributions. We assume that Eq. (1.1) is $\mathbf{U}(1)$-invariant; that is,

$$
F\left(e^{i \theta} \zeta\right)=e^{i \theta} F(\zeta), \quad \zeta \in \mathbb{C}^{2}, \quad \theta \in \mathbb{R}
$$

This condition leads to the existence of two-frequency solitary wave solutions of type

$$
\psi(x, t)=\phi_{\omega_{1}}(x) e^{-i \omega_{1} t}+\phi_{\omega_{2}}(x) e^{-i \omega_{2} t}, \quad\left(\omega_{1}, \omega_{2}\right) \in \mathbb{R}^{2} .
$$

We prove that indeed they form the global attractor for all finite energy solutions to (1.1). Namely, our main result is the following long-time asymptotics: In the case when polynomials $F_{j}$ are strictly nonlinear, any solution with initial data from $H^{1}(\mathbb{R}) \otimes \mathbb{C}^{2}$ converges to the set $\mathscr{S}$ of all solitary waves:

$$
\psi(\cdot, t) \longrightarrow \mathscr{S}, \quad t \rightarrow \pm \infty,
$$

where the convergence holds in local $H^{1}$-seminorms.

The asymptotics of type (1.4) was discovered first for linear wave and Klein-Gordon equations with external potential in the scattering theory $[13,14,27,32]$. In this case, the attractor $\mathscr{S}$ consists of the zero solution only, and the asymptotics means well-known local energy decay.

The attraction to the set of all static stationary states with $\omega=0$ was established in [15-24] for a number nonlinear wave problems.

First results on the attraction to the set of all stationary orbits for nonlinear $\mathbf{U}(1)$ invariant Schrödinger equations were obtained in the context of asymptotic stability. This establishes asymptotics of type (1.4) but only for solutions with initial date close to some stationary orbit, proving the existence of a local attractor. This was first done in $[28,29]$, and then developed in $[1,4-6,8,21]$ and other papers.

The global attraction of type (1.4) to the solitary waves was established (i) in [17,20] for 1D Klein-Gordon equations coupled to nonlinear oscillators; (ii) in [18,19] for nD Klein-Gordon and Dirac equations with mean field interaction; (iii) in [25,26] for 3D wave and Klein-Gordon equations with concentrated nonlinearity. The global wellposedness and the global attraction (1.4) for the Dirac equationits with concentrated nonlinearity was not considered previously as well as the attraction to solitary waves with two frequencies.

In previous works $[17,18,20,25,26]$ for the wave and Klein-Gordon fields the Hamilton functionals are bounded from below under appropriate assumptions. In the case of distributed interaction of the Dirac field with a nonlinear oscillator [19] the Hamilton 
functional is not bounded from below, and the global well-posedness is derived from the charge conservation.

In our case with the point interaction the charge conservation is not sufficient since $\psi(0)$ is not well defined for $\psi \in L^{2}(\mathbb{R})$. That's why we suggest a novel model of $1 \mathrm{D}$ Dirac equation with a nonlinear point interaction (1.1) providing the Hamilton structure and strong a priori estimates.

Let us comment on our approach. First we prove the omega-limit compactness. This means that for each sequence $s_{j} \rightarrow \infty$ the solutions $\psi\left(x, t+s_{j}\right)$ contain an infinite subsequence which converges in energy seminorms for $|x|<R$ and $|t|<T$ for any $R, T>0$. Any limit function is called as the omega-limiting trajectory $\gamma(x, t)$. To prove the global convergence (1.4) is suffices to show that any omega-limiting trajectory lies on $\mathscr{S}$.

The proof relies on the study of the Fourier transform in time $\tilde{\psi}(x, \omega)$ for each $x \in \mathbb{R}$ and of its support supp $\psi(x, \cdot)$ which is the time-spectrum. The key role is played by the absolute continuity of the spectral densities $\tilde{\psi}(x, \cdot)$ outside the spectral gap $[-m, m]$ for each $x \in \mathbb{R}$. The absolute continuity is a nonlinear version of Kato's theorem on the absence of the embedded eigenvalues and provides the dispersion decay for the high energy component. Any omega-limit trajectory is the solution to (1.1).

This absolute continuity provides that the time-spectrum of $\tilde{\gamma}(x, \cdot)=\left(\tilde{\gamma}_{1}(x, \cdot)\right.$, $\left.\tilde{\gamma}_{2}(x, \cdot)\right)$ is contained in the spectral gap $[-m, m]$ for each $x \in \mathbb{R}$. Finally, we apply the Titchmarsh convolution theorem (see [12, Theorem 4.3.3]) to conclude that time-spectrum of each components $\gamma_{j}(x, \cdot)$ of omega-limit trajectory consists of two frequencies. The Titchmarsh theorem controls the inflation of spectrum by the nonlinearity. Physically, these arguments justify the following binary mechanism of the energy radiation, which is responsible for the attraction to the solitary waves: (i) nonlinear energy transfer from the lower to higher harmonics, and (ii) subsequent dispersion decay caused by the energy radiation to infinity.

The general scheme of the proof bring to mind the approach of [17]. Nevertheless the formulation of the problem and the techniques used are not a straightforward generalization of the one-dimensional result [17].

In [17] the problem reduces to proving a global attraction for the solution $\psi_{S}(x, t)$ to the Klein-Gordon equation with the source $F(\psi(0, t)) \delta(x)$ and with zero initial data. In this case the corresponding Fourier transform of $\psi_{S}(x, t)$ has a simple structure. Namely,

$$
\hat{\psi}_{S}(x, \omega)=\hat{z}(\omega) e^{i k(\omega)|x|}, \quad \hat{z}(\omega)=-\hat{f}(\omega) /(2 i k(\omega)), \quad k(\omega)=\sqrt{\omega^{2}-m^{2}}, \quad \omega \in R,
$$

where $f(t)=F(\psi(0, t))$. Moreover, $z(t)=\psi_{S}(0, t)$, and similar representation holds for bounded and dispersion parts of $\psi_{S}(x, t)$. The key role in the proof plays the absolute continuity of $\hat{z}(\omega)$ on the continuous spectrum $|\omega|>m$ of the Klein-Gordon generator.

In our case one need to prove a global attraction for the solution $\psi_{S}(x, t)$ to the Dirac equation with the source $D_{m}^{-1} F(\psi(0, t)) \delta(x)$. The corresponding Fourier transform $\hat{\psi}_{S}(x, \omega)$ has more complicated structure than (1.5) [see formulas (4.3)-(4.4)]. Now $\hat{z}^{ \pm}(\omega) e^{i k(\omega)|x|}$ with $\hat{z}^{ \pm}(\omega)=-\left(I+\frac{m}{\omega} \beta \pm \frac{i k(\omega)}{\omega} \alpha\right) \frac{\hat{f}(\omega)}{2 i k(\omega)}$ is only a part of $\hat{\psi}_{S}(x, \omega)$ for $\pm x>0$. Moreover, $z^{ \pm}(t) \not \equiv \psi_{S}(0, t)$, and the representation (4.4) holds only for $|\omega|>m$. To solve this difficulty, we derive a novel continuity properties for $\hat{f}(\omega)$ (see Lemma 4.3), Moreover, we use an alternative representation (3.14) for $|\omega|<m$. 
The plan of the paper is as follows. In Sect. 2 we state the main assumptions and results. In Sect. 3 we eliminate a dispersive component of the solution and construct spectral representation for the remaining part. In Sect. 4 we prove absolute continuity of high frequency spectrum of the remaining part. In Sect. 5 we exclude the second dispersive component corresponding to the high frequencies. In Sect. 6 we establish compactness for the remaining component with the bounded spectrum. In Sect. 7 we state the spectral properties of all omega-limit trajectories and apply the Titchmarsh Convolution Theorem. In Appendices we establish the global well-posedness for Eq. (1.1) and prove global attraction (1.4) in the case on linear $F(\psi)$.

\section{Main Results}

Model. We consider the Cauchy problem for the Dirac equation coupled to a nonlinear oscillator:

$$
\left\{\begin{array}{l}
i \dot{\psi}(x, t)=D_{m} \psi(x, t)-D_{m}^{-1} \delta(x) F(\psi(0, t)), \quad t \in \mathbb{R} \\
\left.\psi\right|_{t=0}=\psi_{0}(x),
\end{array} \quad x \in \mathbb{R} .\right.
$$

We will assume that the nonlinearity $F=\left(F_{1}, F_{2}\right)$ admits a real-valued potential:

$$
F_{j}(\zeta)=-\partial_{\bar{\zeta}_{j}} U(\zeta), \quad \zeta_{j} \in \mathbb{C}, \quad j=1,2, \quad U \in C^{2}\left(\mathbb{C}^{2}\right)
$$

Then Eq. (2.1) formally can be written as a Hamiltonian system,

$$
\dot{\psi}(t)=J D \mathscr{H}(\psi), \quad J=-i D_{m}^{-1},
$$

where $D \mathscr{H}$ is the variational derivative of the Hamilton functional

$$
\mathscr{H}(\psi)=\frac{1}{2}\left\langle\psi,\left(-\partial_{x}^{2}+m^{2}\right) \psi\right\rangle+U(\psi(0)) .
$$

Global well-posedness. To have a priori estimates available for the proof of the global well-posedness, we assume that

$$
U(\zeta) \geq A-B|\zeta|^{2}, \quad \zeta \in \mathbb{C}^{2}, \quad A \in \mathbb{R}, \quad 0 \leq B<m .
$$

We will write $L^{2}$ and $H^{1}$ instead of $L^{2}(\mathbb{R}) \otimes \mathbb{C}^{2}$ and instead of $H^{1}(\mathbb{R}) \otimes \mathbb{C}^{2}$, respectively.

Theorem 2.1. Let conditions (2.2) and (2.4) hold. Then:

1. For every $\psi_{0} \in H^{1}$ the Cauchy problem (2.1) has a unique solution $\psi(t) \in C\left(\mathbb{R}, H^{1}\right)$ $\cap C^{1}\left(\mathbb{R}, L^{2}\right)$.

2. The map $W(t): \psi_{0} \mapsto \psi(t)$ is continuous in $H^{1}$ for each $t \in \mathbb{R}$.

3. The energy is conserved:

$$
\mathscr{H}(\psi(t))=\text { const }, \quad t \in \mathbb{R} .
$$

4. The following a priori bound holds:

$$
\|\psi(t)\|_{H^{1}} \leq C\left(\psi_{0}\right), \quad t \in \mathbb{R} .
$$

We prove this theorem in "Appendix A". 
Solitary waves and the main theorem. We assume that the nonlinearity is polynomial. More precisely,

$$
U(\zeta)=U_{1}\left(\zeta_{1}\right)+U_{2}\left(\zeta_{2}\right), \quad \zeta_{j} \in \mathbb{C}
$$

where

$$
U_{j}\left(\zeta_{j}\right)=\sum_{n=0}^{N_{j}} u_{n, j}\left|\zeta_{j}\right|^{2 n}, \quad u_{n, j} \in \mathbb{R}, \quad u_{N_{j}, j}>0, \quad N_{j} \geq 2, \quad j=1,2
$$

This assumption guarantees the bound (2.4) and it is crucial in our argument: it allow to apply the Titchmarsh convolution theorem. Equality (2.8) implies that

$$
\begin{aligned}
& F_{j}\left(\zeta_{j}\right)=-\partial_{\bar{\zeta}_{j}} U_{j}\left(\zeta_{j}\right)=a_{j}\left(\left|\zeta_{j}\right|^{2}\right) \zeta_{j}, \quad j=1,2 \\
& a_{j}\left(\left|\zeta_{j}\right|^{2}\right):=-\sum_{n=1}^{N_{j}} 2 n u_{n, j}\left|\zeta_{j}\right|^{2 n-2}
\end{aligned}
$$

Definition 2.2. (i) The solitary wave solution of Eq. (1.1) are solutions of the form

$$
\begin{aligned}
\psi(x, t)= & \phi_{\omega_{1}}(x) e^{-i \omega_{1} t}+\phi_{\omega_{2}}(x) e^{-i \omega_{2} t}, \quad\left(\omega_{1}, \omega_{2}\right) \in \mathbb{R}^{2}, \\
& \phi_{\omega_{k}} \in H^{1}, \quad k=1,2 .
\end{aligned}
$$

(ii) The solitary manifold is the set: $\mathscr{S}=\left\{\phi_{\omega_{1}}+\phi_{\omega_{2}}:\left(\omega_{1}, \omega_{2}\right) \in \mathbb{R}^{2}\right\}$.

Note that for any $\left(\omega_{1}, \omega_{2}\right) \in \mathbb{R}^{2}$ there is a zero solitary wave with $\phi_{\omega_{1}}=\phi_{\omega_{2}} \equiv 0$, since $F(0)=0$. From (2.7) it follows that the set $\mathscr{S}$ is invariant under multiplication by $e^{i \theta}$, $\theta \in \mathbb{R}$.

Denote $\varkappa_{j}=\varkappa\left(\omega_{j}\right)=\sqrt{m^{2}-\omega_{j}^{2}}>0$ for $\omega_{j} \in(-m, m)$.

Proposition 2.3 (Existence of nonzero solitary waves). Assume that $F(\zeta)$ satisfies (2.9). Then nonzero solitary waves may exist only for $\omega_{j} \in(-m, m)$. The amplitudes of solitary waves are given by

$$
\begin{aligned}
& \phi_{\omega_{1}}(x)=C_{1}\left(\begin{array}{c}
e^{-\varkappa_{1}|x|}+\frac{m e^{-\varkappa_{1}|x|}-\varkappa_{1} e^{-m|x|}}{\omega_{1}} \\
\varkappa_{1} \operatorname{sgn} x \frac{e^{-\varkappa_{1}|x|}-e^{-m|x|}}{\omega_{1}}
\end{array}\right), \\
& \phi_{\omega_{2}}(x)=C_{2}\left(\begin{array}{c}
-\varkappa_{2} \operatorname{sgn} x \frac{e^{-\varkappa_{2}|x|}-e^{-m|x|}}{\omega_{2}} \\
e^{-\varkappa_{2}|x|}-\frac{m e^{-\varkappa_{2}|x|}-\varkappa_{2} e^{-m|x|}}{\omega_{2}}
\end{array}\right) .
\end{aligned}
$$

where $C_{j}$ are solutions to

$$
2 C_{j} \varkappa_{j}=F_{j}\left(C_{j}\left[1+(-1)^{j+1} \frac{m-\varkappa\left(\omega_{j}\right)}{\omega_{j}}\right]\right), \quad j=1,2 .
$$


Corollary 2.4. Substituting (2.12) into (2.11) we obtain the following representation for solitary wave solutions

$$
\begin{aligned}
\psi(x, t)= & C_{1}\left(\begin{array}{c}
e^{-\varkappa_{1}|x|}+\frac{m e^{-\varkappa_{1}|x|}-\varkappa_{1} e^{-m|x|}}{\omega_{1}} \\
\varkappa_{1} \operatorname{sgn} x \frac{e^{-\varkappa_{1}|x|}-e^{-m|x|}}{\omega_{1}}
\end{array}\right) e^{-i \omega_{1} t} \\
& +C_{2}\left(\begin{array}{c}
-\varkappa_{2} \operatorname{sgn} x \frac{e^{-\varkappa_{2}|x|}-e^{-m|x|}}{\omega_{2}} \\
e^{-\varkappa_{2}|x|}-\frac{m e^{-\varkappa_{2}|x|}-\varkappa_{2} e^{-m|x|}}{\omega_{2}}
\end{array}\right) e^{-i \omega_{2} t} .
\end{aligned}
$$

Proof of Proposition 2.3. We look for solution $\psi(x, t)$ to (1.1) in the form (2.11). Consider the function

$$
\chi(x, t):=\psi(x, t)-i D_{m}^{-1} \dot{\psi}(x, t)=\chi_{\omega_{1}}(x) e^{-i \omega_{1} t}+\chi_{\omega_{2}}(x) e^{-i \omega_{2} t},
$$

where

$$
\chi_{\omega_{k}}=\phi_{\omega_{k}}-\omega_{k} D_{m}^{-1} \phi_{\omega_{k}}=D_{m}^{-1}\left(D_{m}-\omega_{k}\right) \phi_{\omega_{k}}, \quad k=1,2
$$

Hence,

$$
\begin{aligned}
\phi_{\omega_{k}} & =D_{m}\left(D_{m}-\omega_{k}\right)^{-1} \chi_{\omega_{k}}=D_{m}\left(D_{m}+\omega_{k}\right)\left(D_{m}^{2}-\omega_{k}^{2}\right)^{-1} \chi_{\omega_{k}} \\
& =\chi_{\omega_{k}}+\left(\omega_{k}^{2}+\omega_{k} D_{m}\right)\left(D_{m}^{2}-\omega_{k}^{2}\right)^{-1} \chi_{\omega_{k}} .
\end{aligned}
$$

Equation (1.1) implies, that

$$
D_{m} \chi(x, t)=D_{m} \psi(x, t)-i \dot{\psi}(x, t)=D_{m}^{-1} F(\psi(0, t)) \delta(x) .
$$

Applying the operator $D_{m}$, we obtain by (2.15)

$$
e^{-i \omega_{1} t} D_{m}^{2} \chi_{\omega_{1}}(x)+e^{-i \omega_{2} t} D_{m}^{2} \chi_{\omega_{2}}(x)=F(\psi(0, t)) \delta(x), \quad D_{m}^{2}=-\partial_{x}^{2}+m^{2} .
$$

Therefore, in the case $\omega_{1} \neq \omega_{2}$,

$$
\chi_{\omega_{k}, j}(x)=C_{k j} \frac{e^{-m|x|}}{2 m}, \quad k, j=1,2,
$$

where $C_{k j}$ are solutions to

$$
e^{-i \omega_{1} t} C_{1 j}+e^{-i \omega_{2} t} C_{2 j}=F_{j}\left(\phi_{\omega_{1}, j}(0) e^{-i \omega_{1} t}+\phi_{\omega_{2}, j}(0) e^{-i \omega_{2} t}\right), \quad j=1,2 .
$$

We can also assume this formulas in the case $\omega_{1}=\omega_{2}$ setting $\chi_{\omega_{2}}=0$. We will return to Eq. (2.18) later. First we derive the explicit formulas for $\phi_{\omega_{k}}(x)$, using (2.16) and (2.17) only. Applying [10, Formula 1.2.(11)], we get

$$
\begin{aligned}
\left(D_{m}^{2}-\omega_{k}^{2}\right)^{-1} \frac{C_{k j} e^{-m|x|}}{2 m} & =\frac{C_{k j}}{2 \pi} \int_{\mathbb{R}} \frac{e^{-i \xi x} d \xi}{\left(\xi^{2}+m^{2}\right)\left(\xi^{2}+m^{2}-\omega_{k}^{2}\right)} \\
& =\frac{C_{k j}}{\omega_{k}^{2} \pi} \int_{0}^{\infty}\left(\frac{\cos \xi x}{\xi^{2}+m^{2}-\omega_{k}^{2}}-\frac{\cos \xi x}{\xi^{2}+m^{2}}\right) d \xi \\
& =\frac{C_{k j}}{2 \omega_{k}^{2}}\left(\frac{e^{-\varkappa_{k}|x|}}{\varkappa_{k}}-\frac{e^{-m|x|}}{m}\right), \quad \varkappa_{k}=\varkappa\left(\omega_{k}\right) .
\end{aligned}
$$


Substituting this into (2.16), we obtain

$$
\begin{gathered}
\left(\begin{array}{l}
\phi_{\omega_{k}, 1}(x) \\
\phi_{\omega_{k}, 2}(x)
\end{array}\right)=\left(\begin{array}{l}
C_{k 1} \\
C_{k 2}
\end{array}\right) \frac{e^{-\varkappa_{k}|x|}}{2 \varkappa_{k}}+\left(\begin{array}{c}
C_{k 1} \\
-C_{k 2}
\end{array}\right)\left(\frac{m e^{-\varkappa_{k}|x|}-\varkappa_{k} e^{-m|x|}}{2 \omega_{k} \varkappa_{k}}\right) \\
+\left(\begin{array}{c}
-C_{k 2} \\
C_{k 1}
\end{array}\right) \operatorname{sgn} x \frac{e^{-\varkappa_{k}|x|}-e^{-m|x|}}{2 \omega_{k}} .
\end{gathered}
$$

Hence,

$$
\phi_{\omega_{k}, j}(0)=\frac{C_{k j}}{2 \varkappa_{k}}\left(1+(-1)^{j+1} \frac{m-\varkappa_{k}}{\omega_{k}}\right), \quad k, j=1,2 .
$$

Now we turn to the study of the Eq. (2.18). First, consider the case when $\omega_{1}=\omega_{2}=\omega$. We set $C_{1 j}=C_{j}, C_{2 j}=0, j=1,2$, and Eq. (2.18) becomes

$$
e^{-i \omega t} C_{j}=F_{j}\left(\phi_{\omega, j}(0) e^{-i \omega t}\right)=a_{j}\left(\left|\phi_{\omega, j}(0) e^{-i \omega t}\right|\right) \phi_{\omega, j}(0) e^{-i \omega t}, \quad j=1,2
$$

by (2.9). Using (2.20), we get after cancelation of exponential

$$
2 C_{j}^{\prime} \varkappa=F_{j}\left(\phi_{\omega, j}(0)\right)=F_{j}\left(C_{j}^{\prime}\left(1+(-1)^{j+1} \frac{m-\varkappa}{\omega}\right)\right), \quad \varkappa=\sqrt{m^{2}-\omega^{2}}, \quad j=1,2 .
$$

Here we denote $C_{j}^{\prime}=C_{j} /(2 \varkappa)$. Finally, in the case $\omega_{1}=\omega_{2}=\omega$, Eq. (2.11) reads

$$
\psi(x, t)=\phi_{\omega}(x, t) e^{-i \omega t},
$$

where, in accordance with (2.19),

$$
\left\{\begin{array}{l}
\phi_{\omega, 1}(x)=C_{1}^{\prime}\left(e^{-\varkappa|x|}+\frac{m e^{-\varkappa|x|}-\varkappa e^{-m|x|}}{\omega}\right)-C_{2}^{\prime} \varkappa \operatorname{sgn} x \frac{e^{-\varkappa|x|}-e^{-m|x|}}{2 \omega_{k}} \\
\phi_{\omega, 2}(x)=C_{2}^{\prime}\left(e^{-\varkappa|x|}-\frac{m e^{-\varkappa|x|}-\varkappa e^{-m|x|}}{\omega}\right)+C_{1}^{\prime} \varkappa \operatorname{sgn} x \frac{e^{-\varkappa|x|}-e^{-m|x|}}{2 \omega_{k}}
\end{array} \mid\right.
$$

Now consider the case when $\omega_{1} \neq \omega_{2}$. Taking into account (2.9), we rewrite (2.18) as

$$
\begin{aligned}
e^{-i \omega_{1} t} C_{1 j}+e^{-i \omega_{2} t} C_{2 j}= & a_{j}\left(\left|\phi_{\omega_{1}, j}(0) e^{-i \omega_{1} t}+\phi_{\omega_{2}, j}(0) e^{-i \omega_{2} t}\right|^{2}\right)\left(\phi_{\omega_{1}, j}(0) e^{-i \omega_{1} t}\right. \\
& \left.+\phi_{\omega_{2}, j}(0) e^{-i \omega_{2} t}\right), \quad j=1,2
\end{aligned}
$$

Lemma 2.5. Let $\omega_{1} \neq \omega_{2}$. Then for solutions to (2.18) we have either $\phi_{\omega_{1}, j}(0)=0$ or $\phi_{\omega_{2}, j}(0)=0$ for each $j=1,2$.

Proof. It suffices to consider the case $j=1$ and $\omega_{1}<\omega_{2}$ only. Denote $q_{1}:=\phi_{\omega_{1}, 1}(0)$, $q_{2}:=\phi_{\omega_{2}, 1}(0)$. We should prove that either $q_{1}=0$ or $q_{2}=0$. Assume, to the contrary, that $q_{1} \neq 0$ and $q_{2} \neq 0$. Then

$$
\begin{aligned}
& \left|\phi_{\omega_{1}, 1}(0) e^{-i \omega_{1} t}+\phi_{\omega_{2}, 1}(0) e^{-i \omega_{2} t}\right|^{2}=\left|q_{1}\right|^{2}+\left|q_{2}\right|^{2}+q_{1} \bar{q}_{2} e^{i \delta t}+\bar{q}_{1} q_{2} e^{-i \delta t}, \\
& \quad \delta:=\omega_{2}-\omega_{1}>0,
\end{aligned}
$$

where $q_{1} \bar{q}_{2} \neq 0$ and $\bar{q}_{1} q_{2} \neq 0$. Hence, (2.10) implies

$a_{1}\left(\left|\phi_{\omega_{1}, 1}(0) e^{-i \omega_{1} t}+\phi_{\omega_{2}, 1}(0) e^{-i \omega_{2} t}\right|^{2}\right)=b e^{i\left(N_{j}-1\right) \delta t}+\bar{b} e^{-i\left(N_{j}-1\right) \delta t}+\sum_{|n| \leq N_{1}-2} c_{n} e^{i n \delta t}$, 
where $b \neq 0$ since $a_{1}$ is a polynomial of degree $N_{1}-1 \geq 1$ due to (2.8) and (2.10). Now the right hand side of (2.18) contains the terms $e^{-i\left[\omega_{1} \bar{t}-\left(N_{j}-1\right) \delta\right] t}$ and $e^{-i\left[\omega_{2} t+\left(N_{j}-1\right) \delta\right] t}$ with nonzero coefficients, which are absent on the left hand side. This contradiction proves the lemma.

This lemma and (2.20) imply

Corollary 2.6. Let $\omega_{1} \neq \omega_{2}$. Then for solutions to (2.18) we have either $C_{1, j}=0$ or $C_{2, j}=0$ for each $j=1,2$.

Note that the cases $C_{21}=C_{22}=0$ and $C_{11}=C_{12}=0$ are exactly the case when $\omega_{1}=\omega_{2}$.

Suppose now, that $C_{12}=C_{21}=0$. Then (2.11) and (2.19) imply

$$
\begin{aligned}
\left(\begin{array}{l}
\psi_{1}(x, t) \\
\psi_{2}(x, t)
\end{array}\right) & =\frac{C_{1}}{2 \varkappa_{1}}\left(\begin{array}{c}
e^{-\varkappa_{1}|x|}+\frac{m e^{-\varkappa_{1}|x|}-\varkappa_{1} e^{-m|x|}}{\omega_{1}} \\
\varkappa_{1} \operatorname{sgn} x \frac{e^{-\varkappa_{1}|x|}-e^{-m|x|}}{\omega_{1}}
\end{array}\right) e^{-i \omega_{1} t} \\
& +\frac{C_{2}}{2 \varkappa_{2}}\left(\begin{array}{c}
-\varkappa_{2} \operatorname{sgn} x \frac{e^{-\varkappa_{2}|x|}-e^{-m|x|}}{\omega_{2}} \\
e^{-\varkappa_{2}|x|}-\frac{m e^{-\varkappa_{2}|x|}-\varkappa_{2} e^{-m|x|}}{\omega_{2}}
\end{array}\right) e^{-i \omega_{2} t},
\end{aligned}
$$

with $C_{1}=C_{11}, C_{2}=C_{22}$. Taking into account (2.20), we obtain equations for $C_{j}$ :

$$
C_{j}=F_{j}\left(\frac{C_{j}}{2 \varkappa_{j}}\left[1+(-1)^{j+1} \frac{m-\varkappa_{j}}{\omega_{j}}\right]\right), \quad j=1,2 .
$$

Equations (2.24) and (2.25) will coincide with Eqs. (2.14) and (2.13) after the replacement $C_{j}$ by $2 C_{j} \varkappa_{j}$.

It is easy to check that in the case $C_{11}=C_{22}=0$, we obtain the same formulas, interchanging $\omega_{1}$ and $\omega_{2}$.

Proposition is completely proved.

The following lemma gives a sufficient condition for the existence of nonzero solitary waves.

Lemma 2.7. Let $F$ satisfies (2.9)-(2.10) with $M_{j}=-u_{1, j}>0$, where $j \in\{1 ; 2\}$. Then there exists open subset $I\left(M_{j}\right) \subset(-m, m)$ such that for any $\omega_{j} \in I\left(M_{j}\right)$ the $j$-th equation of (2.13) has nonzero solutions $C_{j}=C_{j}\left(\omega_{j}\right)$. Moreover, if $M_{j}>(1+\sqrt{2}) m$, then $I\left(M_{j}\right)=(-m, m)$.

We prove this lemma in "Appendix C".

Remark 2.8. (i) Equation (2.13) has generally discrete set of solutions $C_{j}$, while $\omega_{j}$ belongs generally to an open set,

(ii) In the linear case, when $F_{j}\left(\psi_{k}\right)=a_{j} \psi_{j}$ with $a_{j} \in \mathbb{R}$, the situation is contrary : we see from (2.13) that

$$
2 \varkappa_{j}=a_{j}\left(1+(-1)^{j+1} \frac{m-\varkappa_{j}}{\omega_{j}}\right), \quad \varkappa_{j}=\sqrt{m^{2}-\omega_{j}^{2}},
$$

i.e., $\omega_{j}$ generally belongs to a discrete set, while $C_{j} \in \mathbb{C}$ is arbitrary.

Our main result is the following theorem.

Theorem 2.9 (Main Theorem). Let the nonlinearity $F(\psi)$ satisfy (2.9)-(2.10). Then for any $\psi_{0} \in H^{1}$ the solution $\psi(t) \in C\left(\mathbb{R}, H^{1}\right)$ to the Cauchy problem (2.1) with $\psi(0)=\psi_{0}$ converges to $\mathscr{S}$ in the space $H_{\text {loc }}^{1}(\mathbb{R}) \otimes C^{2}$ :

$$
\psi(t) \rightarrow \mathscr{S}, \quad t \rightarrow \pm \infty .
$$




\section{Splitting of Solutions}

It suffices to prove Theorem 2.9 for $t \rightarrow+\infty$; We will only consider the solution $\psi(x, t)$ restricted to $t \geq 0$ and split it as

$$
\psi(x, t)=\phi(x, t)+\psi_{S}(x, t), \quad t \geq 0 .
$$

Here $\phi(x, t)$ is a solution to the Cauchy problem for the free Dirac equation

$$
i \dot{\phi}(x, t)=D_{m} \phi(x, t),\left.\quad \phi\right|_{t=0}=\psi_{0},
$$

and $\psi_{S}(x, t)$ is a solution to the Cauchy problem for Dirac equation with the source

$$
i \dot{\psi}_{S}(x, t)=D_{m} \psi_{S}(x, t)-D_{m}^{-1} F(\psi(0, t)) \delta(x), \quad \psi_{S}(x, 0)=0 .
$$

The following lemma states well known local decay for the free Dirac equation.

Lemma 3.1 (cf. [19, Proposition 4.3]). Let $\psi_{0} \in H^{1}$. Then $\phi \in C_{b}\left(\overline{\mathbb{R}^{+}}, H^{1}\right)$, and $\forall R>0$,

$$
\|\phi(t)\|_{H^{1}(-R, R)} \rightarrow 0, \quad t \rightarrow \infty .
$$

Now (2.6) implies that

$$
\psi_{S}=\psi(t)-\phi(t) \in C_{b}\left(\overline{\mathbb{R}^{+}}, H^{1}\right) .
$$

Due to (3.3) it suffices to prove (2.26) for $\psi_{S}$ only.

Complex Fourier-Laplace transform. Let us analyse the complex Fourier-Laplace transform of $\psi_{S}(x, t)$ :

$$
\tilde{\psi}_{S}(x, \omega)=\int_{0}^{\infty} e^{i \omega t} \psi_{S}(x, t) d t, \quad \omega \in \mathbb{C}^{+},
$$

where $\mathbb{C}^{+}:=\{z \in \mathbb{C}: \operatorname{Im} z>0\}$. Due to $(3.4), \tilde{\psi}_{S}(\cdot, \omega)$ is an $H^{1}$-valued analytic function of $\omega \in \mathbb{C}^{+}$.

Denote $f(t)=F\left(\psi(0, t)\right.$. Then Eq. (3.2) for $\psi_{S}$ with zero initial data implies that

$$
\left(D_{m}-\omega\right) \tilde{\psi}_{S}(x, \omega)-D_{m}^{-1} \delta(x) \tilde{f}(\omega)=0, \quad \omega \in \mathbb{C}^{+} .
$$

It is easy to see that

$$
\tilde{\psi}_{S}(x, \omega):=\left(D_{m}+\omega\right) D_{m}^{-1} \tilde{f}(\omega) G(x, \omega)=-\tilde{f}(\omega) G(x, \omega)-\omega D_{m}^{-1} \tilde{f}(\omega) G(x, \omega),
$$

where $G(\cdot, \omega) \in H^{1}$ is the unique elementary solution to

$$
G^{\prime \prime}(x, \omega)+\left(\omega^{2}-m^{2}\right) G(x, \omega)=\delta(x), \quad \omega \in \mathbb{C}^{+} .
$$

This solution is given by $G(x, \omega)=\frac{e^{i k(\omega)|x|}}{2 i k(\omega)}$, where $k(\omega)$ stands for the analytic function

$$
k(\omega):=\sqrt{\omega^{2}-m^{2}}, \quad \operatorname{Im} k(\omega)>0, \quad \omega \in \mathbb{C}^{+},
$$


which we extend to $\omega \in \overline{\mathbb{C}}^{+}$by continuity. Thus,

$$
\tilde{\psi}_{S}(x, \omega)=-\tilde{f}(\omega) \frac{e^{i k(\omega)|x|}}{2 i k(\omega)}-\omega D_{m}^{-1} \tilde{f}(\omega) \frac{e^{i k(\omega)|x|}}{2 i k(\omega)}, \quad \omega \in \mathbb{C}^{+} .
$$

Note, that

$$
D_{m}^{-2} \frac{e^{i k(\omega)|x|}}{2 i k(\omega)}=\frac{1}{2 \pi} \int \frac{e^{-i k x} d k}{\left(k^{2}+m^{2}\right)\left(-k^{2}-m^{2}+\omega^{2}\right)}=\frac{1}{\omega^{2}}\left(\frac{e^{-m|x|}}{2 m}+\frac{e^{i k(\omega)|x|}}{2 i k(\omega)}\right) .
$$

Therefore,

$\omega D_{m}^{-1} \tilde{f}(\omega) \frac{e^{i k(\omega)|x|}}{2 i k(\omega)}=m \beta \frac{\tilde{f}(\omega)}{\omega}\left(\frac{e^{-m|x|}}{2 m}+\frac{e^{i k(\omega)|x|}}{2 i k(\omega)}\right)+\alpha \frac{\tilde{f}(\omega)}{2 \omega} \operatorname{sgn} x\left(e^{i k(\omega)|x|}-e^{-m|x|}\right)$,

and (3.7) becomes

$$
\begin{aligned}
\tilde{\psi}_{S}(x, \omega)= & -\tilde{f}(\omega) \frac{e^{i k(\omega)|x|}}{2 i k(\omega)}-m \beta \frac{\tilde{f}(\omega)}{\omega}\left(\frac{e^{-m|x|}}{2 m}+\frac{e^{i k(\omega)|x|}}{2 i k(\omega)}\right) \\
& -\alpha \frac{\tilde{f}(\omega)}{2 \omega} \operatorname{sgn} x\left(e^{i k(\omega)|x|}-e^{-m|x|}\right)=-\left(I+\beta \frac{m+i k(\omega)}{\omega}\right) \frac{\tilde{f}(\omega)}{2 i k(\omega)} e^{i k(\omega)|x|} \\
& +(\beta-\alpha \operatorname{sgn} x) \tilde{f}(\omega) \frac{e^{i k(\omega)|x|}-e^{-m|x|}}{2 \omega}, \quad \omega \in \mathbb{C}^{+} .
\end{aligned}
$$

Here the last term vanishes for $x=0$. Denote $y(t):=\psi_{S}(0, t) \in C_{b}(\mathbb{R})$. Then (3.8) implies

$$
\tilde{y}(\omega)=\tilde{\psi}_{S}(0, \omega)=-\left(I+\beta \frac{m+i k(\omega)}{\omega}\right) \frac{\tilde{f}(\omega)}{2 i k(\omega)}, \quad \omega \in \mathbb{C}^{+} .
$$

Now (3.8) becomes

$$
\tilde{\psi}_{S}(x, \omega)=\tilde{y}(\omega) e^{i k(\omega)|x|}+(\beta-\alpha \operatorname{sgn} x) \tilde{f}(\omega) \frac{e^{i k(\omega)|x|}-e^{-m|x|}}{2 \omega}, \quad \omega \in \mathbb{C}^{+} .
$$

Let us extend $\psi_{S}(x, t)$ and $f(t)$ by zero for $t<0$. Then

$$
\psi_{S} \in C_{b}\left(\mathbb{R}, H^{1}\right)
$$

by (3.4). The Fourier transform $\hat{\psi}_{S}(\cdot, \omega):=\mathscr{F}_{t \rightarrow \omega}\left[\psi_{S}(\cdot, t)\right]$ is a tempered $H^{1}$-valued distribution of $\omega \in \mathbb{R}$. The distribution $\hat{\psi}_{S}(\cdot, \omega)$ is the boundary value of the analytic $H^{1}$-valued function $\tilde{\psi}_{S}(\cdot, \omega)$, in the following sense:

$$
\hat{\psi}_{S}(\cdot, \omega)=\lim _{\varepsilon \rightarrow 0+} \tilde{\psi}_{S}(\cdot, \omega+i \varepsilon), \quad \omega \in \mathbb{R},
$$

where the convergence holds in the space of tempered distributions $\mathscr{S}^{\prime}\left(\mathbb{R}, H^{1}\right)$. Indeed,

$$
\tilde{\psi}_{S}(\cdot, \omega+i \varepsilon)=\mathscr{F}_{t \rightarrow \omega}\left[\psi_{S}(\cdot, t) e^{-\varepsilon t}\right],
$$

and $\psi_{S}(\cdot, t) e^{-\varepsilon t} \underset{\varepsilon \rightarrow 0+}{\longrightarrow} \psi_{S}(\cdot, t)$, where the convergence holds in $\mathscr{S}^{\prime}\left(\mathbb{R}, H^{1}\right)$ by (3.11). Therefore, (3.12) holds by the continuity of the Fourier transform $\mathscr{F}_{t \rightarrow \omega}$ in $\mathscr{S}^{\prime}(\mathbb{R})$. 
Similarly to (3.12), the distributions $\hat{f}(\omega)$ and $\hat{y}(\omega)$ of $\omega \in \mathbb{R}$ are boundary values of analytic in $\mathbb{C}^{+}$functions $\tilde{f}(\omega)$ and $\tilde{y}(\omega), \omega \in \mathbb{C}^{+}$, respectively:

$$
\begin{aligned}
& \hat{f}(\omega)=\lim _{\varepsilon \rightarrow 0+} \tilde{f}(\omega+i \varepsilon) \\
& \hat{y}(\omega)=\lim _{\varepsilon \rightarrow 0+} \tilde{y}(\omega+i \varepsilon)=-\left(I+\beta \frac{m+i k(\omega)}{\omega}\right) \frac{\hat{f}(\omega)}{2 i k(\omega)}
\end{aligned} \mid \omega \in \mathbb{R},
$$

since the function $f(t)=F(\psi(0, t))$ is bounded for $t \geq 0$ and vanishes for $t<0$. The convergences hold in the space of tempered distributions $\mathscr{S}^{\prime}(\mathbb{R})$. Let us justify that the representation (3.10) for $\hat{\psi}_{S}(x, \omega)$ is also valid when $\omega \in \mathbb{R}$.

Lemma 3.2. For any fixed $x \in \mathbb{R}$,

$$
\hat{\psi}_{S}(x, \omega)=\hat{y}(\omega) e^{i k(\omega)|x|}+(\beta-\alpha \operatorname{sgn} x) \hat{f}(\omega) \frac{e^{i k(\omega)|x|}-e^{-m|x|}}{2 \omega}, \quad \omega \in \mathbb{R}
$$

Here the multiplications are understood in the sense of quasimeasures (see [17, Appendix B]).

The proof follows from (3.10) similarly to [17, Proposition 3.1]. Namely, the convergence (3.13) holds in the space of quasimeasures, while $e^{i k(\omega)|x|}$ and $\frac{e^{i k(\omega)|x|}-e^{-m|x|}}{2 \omega}$ are multiplicators in the space of quasimeasures.

\section{Absolutely Continuous Spectrum}

Denote

$$
\Omega_{\delta}:=(-\infty,-m-\delta) \cup(m+\delta, \infty), \quad \delta \geq 0 .
$$

Consider the functions

$$
\tilde{z}^{ \pm}(\omega):=-\left(I+\frac{m}{\omega} \beta \pm \frac{i k(\omega)}{\omega} \alpha\right) \frac{\tilde{f}(\omega)}{2 i k(\omega)}, \quad \omega \in \mathbb{C}^{+} .
$$

From (3.13) it follows that for $\omega \in \Omega_{0}$ there exist

$$
\lim _{\varepsilon \rightarrow 0+} \frac{\tilde{f}(\omega+i \varepsilon)}{\omega+i \varepsilon}=\frac{\hat{f}(\omega)}{\omega}, \quad \lim _{\varepsilon \rightarrow 0+}\left(I+\beta \frac{m}{\omega+i \varepsilon}\right) \frac{\tilde{f}(\omega+i \varepsilon)}{2 i k(\omega+i \varepsilon)}=\left(I+\beta \frac{m}{\omega}\right) \frac{\hat{f}(\omega)}{2 i k(\omega)} .
$$

Hence, for $\omega \in \Omega_{0}$ there exist boundary values $\hat{z}^{ \pm}(\omega)$ of $\tilde{z}^{ \pm}(\omega)$ :

$$
\hat{z}^{ \pm}(\omega)=\lim _{\varepsilon \rightarrow 0+} \tilde{z}^{ \pm}(\omega+i \varepsilon)=-\left(I+\frac{m}{\omega} \beta \pm \frac{i k(\omega)}{\omega} \alpha\right) \frac{\hat{f}(\omega)}{2 i k(\omega)}, \quad \omega \in \Omega_{0} .
$$

Now we rewrite (3.10) as

$$
\hat{\psi}_{S}(x, \omega)=e^{i k(\omega)|x|} \hat{z}^{ \pm}(\omega)+e^{-m|x|}( \pm \alpha-\beta) \frac{\hat{f}(\omega)}{2 \omega}, \quad \pm x>0, \quad \omega \in \Omega_{0} .
$$

We study the regularity of $\hat{z}^{ \pm}(\omega)$. Note that the function $\omega k(\omega)$ is positive for $\omega \in \Omega_{0}$. 
Proposition 4.1. The distributions $\hat{z}^{ \pm}(\omega)$ are absolutely continuous for $\omega \in \Omega_{0}$, i.e. and $\hat{z}^{ \pm} \in L_{\text {loc }}^{1}\left(\Omega_{0}\right)$. Moreover,

$$
\int_{\Omega_{0}}\left(\left|\hat{z}^{+}(\omega)\right|^{2}+\left|\hat{z}^{-}(\omega)\right|^{2}\right) \omega k(\omega) d \omega<\infty .
$$

Proof. We use the arguments of Paley-Wiener type. Namely, the Parseval identity and (3.4) imply that

$$
\int_{\mathbb{R}}\left\|\tilde{\psi}_{S}(\cdot, \omega+i \varepsilon)\right\|_{H^{1}}^{2} d \omega=2 \pi \int_{0}^{\infty} e^{-2 \varepsilon t}\left\|\psi_{S}(\cdot, t)\right\|_{H^{1}}^{2} d t \leq \frac{C}{\varepsilon}, \quad \varepsilon>0 .
$$

Then (4.6) gives

$$
\int_{\mathbb{R}}\left\|\tilde{\psi}_{S}(\cdot, \omega+i \varepsilon)\right\|_{H^{1}}^{2} d \omega \leq \frac{C}{\varepsilon}, \quad \varepsilon>0 .
$$

Evidently,

$$
\lim _{\varepsilon \rightarrow 0+} \varepsilon\left\|e^{-m|x|}\right\|_{H^{1}} \rightarrow 0 .
$$

Hence, (4.4) and (4.7) results in

$$
\begin{aligned}
\varepsilon \int_{\Omega_{0}}( & \left|\tilde{z}^{+}(\omega+i \varepsilon)\right|^{2}\left\|e^{i k(\omega+i \varepsilon)|x|}\right\|_{H^{1}(0, \infty)}^{2} \\
& \left.+\left|\tilde{z}^{-}(\omega+i \varepsilon)\right|^{2}\left\|e^{i k(\omega+i \varepsilon)|x|}\right\|_{H^{1}(-\infty, 0)}^{2}\right) d \omega \leq C, \quad \varepsilon>0 .
\end{aligned}
$$

Here is a crucial observation about the norm of $e^{i k(\omega+i \varepsilon)|x|}$.

Lemma 4.2 (cf. [17, Lemma 3.2]).

1. For $\omega \in \mathbb{R}$,

$$
\lim _{\varepsilon \rightarrow 0+} \varepsilon\left\|e^{i k(\omega+i \varepsilon)|x|}\right\|_{H^{1}}^{2}=n(\omega):= \begin{cases}\omega k(\omega), & |\omega|>m \\ 0, & |\omega|<m\end{cases}
$$

where the norm in $H^{1}$ is chosen to be $\|\psi\|_{H^{1}}=\left(\left\|\psi^{\prime} \mid\right\|_{L^{2}}^{2}+m^{2}\|\psi\|_{L^{2}}^{2}\right)^{1 / 2}$.

2. For any $\delta>0$ there exists $\varepsilon_{\delta}>0$ such that

$$
\varepsilon\left\|e^{i k(\omega+i \varepsilon)|x|}\right\|_{H^{1}}^{2} \geq n(\omega) / 2, \quad \omega \in \Omega_{\delta}, \quad \varepsilon \in\left(0, \varepsilon_{\delta}\right) .
$$

Substituting (4.10) into (4.8), we get:

$$
\int_{\Omega_{\delta}}\left(\left|\tilde{z}^{+}(\omega+i \varepsilon)\right|^{2}+\left|\tilde{z}^{-}(\omega+i \varepsilon)\right|^{2}\right) \omega k(\omega) d \omega \leq 2 C, \quad 0<\varepsilon<\varepsilon_{\delta},
$$

with the same constant $C$ as in (4.8), and the region $\Omega_{\delta}$ defined in (4.1). We conclude that for each $\delta>0$ the set of functions

$$
g_{\delta, \varepsilon}^{ \pm}(\omega)=\tilde{z}^{ \pm}(\omega+i \varepsilon)|\omega k(\omega)|^{1 / 2}, \quad \varepsilon \in\left(0, \varepsilon_{\delta}\right),
$$


defined for $\omega \in \Omega_{\delta}$, is bounded in the Hilbert space $L^{2}\left(\Omega_{\delta}\right)$, and, by the Banach Theorem, is weakly compact. Hence, the convergence of the distributions (3.13) implies the following weak convergence in the Hilbert space $L^{2}\left(\Omega_{\delta}\right)$ :

$$
g_{\delta, \varepsilon}^{ \pm} \rightarrow g_{\delta}^{ \pm}, \quad \varepsilon \rightarrow 0+,
$$

where the limit function $g_{\delta}^{ \pm}(\omega)$ coincides with the distribution $\hat{z}^{ \pm}(\omega)|\omega k(\omega)|^{1 / 2}$ restricted onto $\Omega_{\delta}$. It remains to note that the norms of all functions $g_{\delta}^{ \pm}, \delta>0$, are bounded in $L^{2}\left(\Omega_{\delta}\right)$ by (4.11), hence (4.5) follows. Finally, $\hat{z}^{ \pm}(\omega) \in L_{\text {loc }}^{1}\left(\Omega_{0}\right)$ by (4.5) and the Cauchy-Schwarz inequality.

\section{Lemma 4.3.}

$$
\int_{\Omega_{0}}|\hat{f}(\omega)|^{2} \frac{k(\omega)}{\omega} d \omega<\infty
$$

Proof. Denote the $2 \times 2$ matrix $A^{ \pm}(\omega)=I+\frac{m}{\omega} \beta \pm \frac{i k(\omega)}{\omega} \alpha$. Then $\hat{z}^{ \pm}(\omega)=-A^{ \pm}(\omega) \frac{\hat{f}(\omega)}{2 i k(\omega)}$. For any $\omega \in \Omega_{0}$, the matrix $A^{ \pm}(\omega)$ has two eigenvalues: $\lambda=0$ and $\lambda=2$ since

$$
\operatorname{det}\left(A^{ \pm}-\lambda I\right)=\left|\begin{array}{cc}
\left(1+\frac{m}{\omega}\right)-\lambda & \pm \frac{i k(\omega)}{\omega} \\
\mp \frac{i k(\omega)}{\omega} & \left(1-\frac{m}{\omega}\right)-\lambda
\end{array}\right|=-2 \lambda+\lambda^{2}=\lambda(\lambda-2) .
$$

The unit eigenvectors $v^{ \pm}(\omega)$ of operators $A^{ \pm}(\omega)$ corresponding to the eigenvalue $\lambda=2$ read

$$
v^{ \pm}(\omega)=\left(\sqrt{\frac{\omega+m}{2 \omega}}, \mp i \sqrt{\frac{\omega-m}{2 \omega}}\right) .
$$

Denote $g(\omega):=\frac{\hat{f}(\omega)}{2 i k(\omega)}$, and $a^{ \pm}(\omega):=\left\langle g(\omega), \nu^{ \pm}(\omega)\right\rangle$. Then $\hat{z}^{ \pm}(\omega)=-A^{ \pm}(\omega) g(\omega)=$ $-2 a^{ \pm}(\omega) v^{ \pm}(\omega)$, and hence

$$
a^{ \pm}(\omega)=\left\langle g(\omega), v^{ \pm}(\omega)\right\rangle=-\frac{1}{2}\left\langle\hat{z}^{ \pm}(\omega), v^{ \pm}(\omega)\right\rangle .
$$

Taking into account (4.14), we get the system of equations for $g(\omega)=\left(g_{1}(\omega), g_{2}(\omega)\right)$

$$
\left\{\begin{array}{c}
g_{1}(\omega) \sqrt{\frac{\omega+m}{2 \omega}}+i g_{2}(\omega) \sqrt{\frac{\omega-m}{2 \omega}}=-\frac{1}{2}\left\langle\hat{z}^{+}(\omega), v^{+}(\omega)\right\rangle \\
g_{1}(\omega) \sqrt{\frac{\omega+m}{2 \omega}}-i g_{2}(\omega) \sqrt{\frac{\omega-m}{2 \omega}}=-\frac{1}{2}\left\langle\hat{z}^{-}(\omega), v^{-}(\omega)\right\rangle
\end{array} \mid .\right.
$$

Therefore

$$
\left(\begin{array}{l}
\hat{f}_{1}(\omega) \\
\hat{f}_{2}(\omega)
\end{array}\right)=2 i k(\omega)\left(\begin{array}{l}
g_{1}(\omega) \\
g_{2}(\omega)
\end{array}\right)=-|\omega|\left(\begin{array}{cc}
i \sqrt{\frac{\omega-m}{2 \omega}} & i \sqrt{\frac{\omega-m}{2 \omega}} \\
\sqrt{\frac{\omega+m}{2 \omega}} & -\sqrt{\frac{\omega+m}{2 \omega}}
\end{array}\right)\left(\begin{array}{c}
\left\langle\hat{z}^{+}(\omega), v^{+}(\omega)\right\rangle \\
\left\langle\hat{z}^{-}(\omega), v^{-}(\omega)\right\rangle
\end{array}\right) .
$$

Hence,

$$
|\hat{f}(\omega)|^{2} \leq C|\omega|^{2}\left(\left|\hat{z}^{+}(\omega)\right|^{2}+\left|\hat{z}^{-}(\omega)\right|^{2}\right) .
$$

Now (4.13) follows from (4.5). 


\section{Further Decomposition of Solutions}

Denote

$$
\hat{f}_{d}(\omega):=\left\{\begin{array}{cc}
\hat{f}(\omega), & \omega \in \Omega_{0} \\
0, & \omega \in \mathbb{R} \backslash \Omega_{0}
\end{array}\right.
$$

and set

$$
\hat{\psi}_{d}(x, \omega)=e^{i k(\omega)|x|} \hat{z}_{d}(x, \omega)+e^{-m|x|}(\alpha \operatorname{sgn} x-\beta) \frac{\hat{f}_{d}(\omega)}{2 \omega}, \quad \omega \in \mathbb{R},
$$

where

$$
\begin{aligned}
\hat{z}_{d}(x, \omega) & =-\left(I+\frac{m}{\omega} \beta+\frac{i k(\omega)}{\omega} \alpha \operatorname{sgn} x\right) \frac{\hat{f}_{d}(\omega)}{2 i k(\omega)} \\
& =-\left(I+\frac{m}{\omega} \beta \pm \frac{i k(\omega)}{\omega} \alpha\right) \frac{\hat{f}_{d}(\omega)}{2 i k(\omega)}=\hat{z}_{d}^{ \pm}(\omega), \quad \pm x>0 .
\end{aligned}
$$

Consider

$$
\psi_{d}(x, t):=\frac{1}{2 \pi} \int_{\mathbb{R}} \hat{\psi}_{d}(x, \omega) e^{-i \omega t} d \omega, \quad x \in \mathbb{R}, \quad t \in \mathbb{R} .
$$

We will show that $\psi_{d}(x, t)$ is a dispersive component of the solution $\psi(x, t)$, in the following sense.

Proposition 5.1. (i) $\psi_{d}(\cdot, t)$ is a bounded continuous $H^{1}$-valued function:

$$
\psi_{d}(\cdot, t) \in C_{b}\left(\mathbb{R}, H^{1}\right) .
$$

(ii) The local energy decay holds for $\psi_{d}(\cdot, t)$ : for any $R>0$,

$$
\left\|\psi_{d}(\cdot, t)\right\|_{H^{1}(-R, R)} \rightarrow 0, \quad t \rightarrow \infty .
$$

Proof. We split $\psi_{d}(x, t)$ as $\psi_{d}(x, t)=\varphi_{d}(x, t)+\chi_{d}(x, t)$, where

$$
\begin{gathered}
\varphi_{d}(x, t)=\frac{1}{2 \pi} \int_{\mathbb{R}} e^{-i \omega t} e^{i k(\omega)|x|} \hat{z}_{d}(x, \omega) d \omega, \\
\chi_{d}(x, t)=\frac{1}{2 \pi} e^{-m|x|}(\alpha \operatorname{sgn} x-\beta) \int_{\mathbb{R}} e^{-i \omega t} \frac{\hat{f}_{d}(\omega)}{2 \omega} d \omega .
\end{gathered}
$$

First, consider $\chi_{d}(x, t)$. Note that

$$
\begin{aligned}
& \int_{\mathbb{R}}\left|\frac{\hat{f}_{d}(\omega)}{\omega}\right| d \omega=\int_{\Omega_{0}}\left|\frac{\hat{f}_{d}(\omega)}{\sqrt{\omega k(\omega)}} \sqrt{\frac{k(\omega)}{\omega}}\right| d \omega \leq\left(\int_{\Omega_{0}}|\hat{f}(\omega)|^{2} \frac{k(\omega)}{\omega} d \omega\right)^{1 / 2} \\
& \quad \times\left(\int_{\Omega_{0}} \frac{d \omega}{\left.\omega \sqrt{\omega^{2}-m^{2}}\right)}\right)^{1 / 2}<\infty
\end{aligned}
$$

by Lemma 4.3. Hence,

$$
\chi_{d} \in C_{b}\left(\mathbb{R}, H^{1}(\mathbb{R} \backslash 0)\right) .
$$


Moreover,

$$
\left\|\chi_{d}(\cdot, t)\right\|_{H^{1}(\mathbb{R} \backslash 0)} \rightarrow 0, \quad t \rightarrow \infty
$$

by Riemann-Lebesgue Theorem. Now consider $\varphi_{d}(x, t)$. Changing the variable $\omega \rightarrow$ $k(\omega)=\sqrt{\omega^{2}-m^{2}}$, we rewrite $\varphi_{d}(x, t)$ as follows:

$$
\varphi_{d}(x, t)=\frac{1}{2 \pi} \int_{\mathbb{R}} \hat{z}_{d}(x, \omega(k)) e^{-i \omega(k) t} e^{i k|x|} \frac{k d k}{\omega(k)} .
$$

Here $\omega(k)=\sqrt{k^{2}+m^{2}}$ is the branch analytic for $\operatorname{Im} k>0$ and continuous for $\operatorname{Im} k \geq 0$. Note that the function $\omega(k), k \in \mathbb{R} \backslash 0$, is the inverse function to $k(\omega)$ defined on $\overline{\mathbb{C}}^{+}$[see (3.6)] and restricted onto $\Omega_{0}$. Let us introduce the functions

$$
\varphi^{ \pm}(x, t)=\frac{1}{2 \pi} \int_{\mathbb{R}} \hat{z}_{d}^{ \pm}(\omega(k)) e^{ \pm i k x} e^{-i \omega(k) t} \frac{k d k}{\omega(k)}, \quad x \in \mathbb{R}, \quad t \geq 0 .
$$

Both functions $\varphi^{ \pm}(x, t)$ are solutions to the free Dirac equation (3.1) on the whole real line (see "Appendix B"). Moreover,

$$
\partial_{x} \varphi^{ \pm}(x, t):=\frac{1}{2 \pi} \int_{\mathbb{R}} \pm i k \hat{z}_{d}^{ \pm}(\omega(k)) e^{ \pm i k x} e^{-i \omega(k) t} \frac{k d k}{\omega(k)}, \quad x \in \mathbb{R}, \quad t \geq 0 .
$$

Hence, the Parseval identity implies

$$
\begin{aligned}
\left\|\varphi^{ \pm}(\cdot, 0)\right\|_{H^{1}}^{2} & =\int_{\mathbb{R}}\left(m^{2}+k^{2}\right)\left|\hat{z}_{d}^{ \pm}(\omega(k))\right|^{2} \frac{k^{2}}{\omega^{2}(k)} d k \\
& =\int_{\Omega_{0}} \omega^{2}\left|\hat{z}^{ \pm}(\omega)\right|^{2} \frac{k(\omega)}{\omega} d \omega=\int_{\Omega_{0}}\left|\hat{z}^{ \pm}(\omega)\right|^{2} \omega k(\omega) d \omega<\infty
\end{aligned}
$$

by (4.11). Hence, both $\varphi^{-}$and $\varphi^{+}$are bounded continuous $H^{1}$-valued functions:

$$
\varphi^{ \pm} \in C_{b}\left(\mathbb{R}, H^{1}\right)
$$

and for any $R>0$

$$
\left\|\varphi^{ \pm}(\cdot, t)\right\|_{H^{1}(-R, R)} \rightarrow 0, \quad t \rightarrow \infty
$$

by Lemma 3.1. The function $\varphi_{d}(x, t)$ coincides with $\varphi^{+}(x, t)$ for $x \geq 0$ and with $\varphi^{-}(x, t)$ for $x \leq 0$ :

$$
\varphi_{d}(x, t)=\varphi^{ \pm}(x, t), \quad \pm x \geq 0 .
$$

It remains to note that $\psi_{d}(x, t)=\varphi_{d}(x, t)+\chi_{d}(x, t)$ has no jump at $x=0$ and therefore $\partial_{x} \psi_{d}(x, t)$ is square-integrable over the whole $x$-axis. Hence,

$$
\left\|\psi_{d}(t)\right\|_{H^{1}}^{2}=\left\|\psi_{d}(t)\right\|_{H^{1}\left(\mathbb{R}^{-}\right)}^{2}+\left\|\psi_{d}(t)\right\|_{H^{1}\left(\mathbb{R}^{+}\right)}^{2} .
$$

Finally, (5.5) follows from (5.13) and (5.9), and (5.6) follows from (5.14) and (5.10).

Denote $y_{d}(t)=\psi_{d}(0, t) \in C_{b}(\mid \mathbb{R})$. Formulas (5.2) and (5.3) imply

$$
\hat{y}_{d}(\omega)=\hat{\psi}_{d}(0, \omega)=-\left(I+\frac{m+i k(\omega)}{\omega} \beta\right) \frac{\hat{f}_{d}(\omega)}{2 i k(\omega)}, \quad \omega \in \mathbb{R},
$$

and (5.2) becomes

$$
\hat{\psi}_{d}(x, \omega)=\hat{y}_{d} e^{-\varkappa(\omega)|x|}+(\beta-\alpha \operatorname{sgn} x) \hat{f}(\omega) \frac{e^{i k(\omega)|x|}-e^{-m|x|}}{2 \omega}, \quad \omega \in \mathbb{R} .
$$




\section{Bound Component}

Spectral representation. We introduce the bound component of the solution $\psi(x, t)$ by

$$
\psi_{b}(x, t)=\psi_{S}(x, t)-\psi_{d}(x, t), \quad x \in \mathbb{R}, \quad t \in \mathbb{R} .
$$

Then (3.11) and (5.5) imply that

$$
\psi_{b} \in C_{b}\left(\mathbb{R}, H^{1}\right)
$$

In particular, $y_{b}(t):=\psi_{b}(0, t)=\psi_{S}(0, t)-\psi_{d}(0, t) \in C_{b}(\mathbb{R})$. Hence, $\hat{y}_{b}(\omega):=$ $\hat{\psi}_{b}(0, \omega)$ is a quasimeasure. Moreover, formulas (3.13) and (5.16) yield

$$
\hat{y}_{b}(\omega)=\hat{y}(\omega)-\hat{y}_{d}(\omega)=-\left(I+\frac{m+i k(\omega)}{\omega} \beta\right) \frac{\hat{f}_{b}(\omega)}{2 i k(\omega)} .
$$

Here we denote

$$
\hat{f}_{b}(\omega):=\hat{f}(\omega)-\hat{f}_{d}(\omega)
$$

Further, (5.1) implies, that

$$
\operatorname{supp} \hat{y}_{b}(\omega)=\operatorname{supp} \hat{\psi}_{b}(0, \omega) \subset[-m, m] .
$$

Denote

$$
\varkappa(\omega):=-i k(\omega)=\sqrt{m^{2}-\omega^{2}}, \quad \operatorname{Re} \varkappa(\omega) \geq 0 \text { for } \operatorname{Im} \omega \geq 0,
$$

where $k(\omega)$ was introduced in (3.6). Let us note that $\varkappa(\omega)>0$ for $\omega \in(-m, m)$. Now we rewrite (6.3) as

$$
\hat{y}_{b}(\omega)=\left(I+\frac{m-\varkappa(\omega)}{\omega} \beta\right) \frac{\hat{f}_{b}(\omega)}{2 \varkappa(\omega)}=\sigma(\omega) \hat{f}_{b}(\omega),
$$

where

$$
\sigma(\omega)=\frac{1}{2 \varkappa(\omega)}\left(\begin{array}{cc}
1+\frac{m-\varkappa(\omega)}{\omega} & 0 \\
0 & 1-\frac{m-\varkappa(\omega)}{\omega}
\end{array}\right)
$$

Hence

$$
\hat{f}_{b}(\omega)=\sigma^{-1}(\omega) \hat{y}_{b}(\omega), \quad \sigma^{-1}(\omega)=\left(\begin{array}{cc}
\varkappa(\omega)+m-\omega & 0 \\
0 & \varkappa(\omega)+m+\omega
\end{array}\right) .
$$

Now (3.14), (5.17), (6.1) and (6.8) imply the multiplicative relation

$$
\hat{\psi}_{b}(x, \omega)=\hat{y}_{b} e^{-\varkappa(\omega)|x|}+\hat{h}_{b}(x, \omega) \frac{e^{-\varkappa(\omega)|x|}-e^{-m|x|}}{2 \omega}, \quad \omega \in \mathbb{R},
$$

where we denote

$$
\hat{h}_{b}(x, \omega)=(\beta-\alpha \operatorname{sgn} x) \sigma^{-1}(\omega) \hat{y}_{b}(\omega) .
$$

From (6.8) and (6.10) it follows that $\hat{h}_{b}(x, \omega)$ for any fixed $x \in \mathbb{R} \backslash 0$ is a quasimeasure with the support $\operatorname{supp}_{b}(x, \omega) \subset[-m, m]$. Moreover, $e^{-\varkappa(\omega)|x|}$ and $\left(e^{-\varkappa(\omega)|x|}-\right.$ $\left.e^{-m|x|}\right) / \omega$ are multiplicators. Hence, function $\hat{\psi}_{b}(x, \omega)$ is quasimeasure for any fixed $x \in \mathbb{R}$ with supports in $[-m, m]$. Finally,

$$
\psi_{b}(x, t)=\frac{1}{2 \pi}\left\langle\hat{\psi}_{b}(x, \omega), e^{-i \omega t}\right\rangle, \quad x \in \mathbb{R}, \quad t \in \mathbb{R},
$$

where $\langle\cdot, \cdot\rangle$ is an extension of the scalar product $\langle f, g\rangle=\int f(\omega) \bar{g}(\omega) d \omega$. 
Compactness. We are going to prove a compactness of the set of translations of the bound component, $\left\{\psi_{b, n}(x, s+t): s \geq 0\right\}, n=1,2$.

Lemma 6.1. (i) The function $\psi_{b}(x, t)$ is smooth for $x \neq 0$ and $t \in \mathbb{R}$. Moreover, for any fixed $x \neq 0, t \in \mathbb{R}$, and any nonnegative integers $j, k$, the following representation holds

$$
\partial_{x}^{j} \partial_{t}^{k} \psi_{b}(x, t)=\frac{1}{2 \pi}\left\langle\Lambda_{j}(x, \omega),(-i \omega)^{k} e^{-i \omega t}\right\rangle
$$

where

$$
\begin{aligned}
\Lambda_{j}(x, \omega)= & (-\varkappa(\omega) \operatorname{sgn} x)^{j} e^{-\varkappa(\omega)|x|} \hat{y}_{b}(\omega) \\
& +\hat{h}_{b}(x, \omega)\left[(-\varkappa(\omega) \operatorname{sgn} x)^{j} \frac{e^{-\varkappa(\omega)|x|}-e^{-m|x|}}{2 \omega}\right. \\
& \left.+\frac{\varkappa^{j}(\omega)-m^{j}}{\omega}(-\operatorname{sgn} x)^{j} e^{-m|x|}\right] .
\end{aligned}
$$

(ii) There is a constant $C_{j, k}>0$ so that

$$
\sup _{x \neq 0} \sup _{t \in \mathbb{R}}\left|\partial_{x}^{j} \partial_{t}^{k} \psi_{b}(x, t)\right| \leq C_{j, k} .
$$

The lemma follows similarly Proposition 4.1 from [17], since the factors $e^{-\varkappa(\omega)|x|} \zeta(\omega)$, $\frac{e^{-\varkappa(\omega)|x|}-e^{-m|x|}}{2 \omega} \zeta(\omega)$, and $\frac{\varkappa^{j}(\omega)-m^{j}}{\omega} \zeta(\omega)$ are multiplicators in the space of quasimeasures. Here $\zeta(\omega) \in C_{0}^{\infty}(\mathbb{R})$ is any cutoff function satisfying

$$
\left.\zeta\right|_{[-m-1, m+1]}=1 \text {. }
$$

Corollary 6.2. By the Ascoli-Arzelà Theorem, for any sequence $s_{l} \rightarrow \infty$ there exists a subsequence (which we also denote by $s_{l}$ ) such that for any nonnegative integers $j$ and $k$,

$$
\partial_{x}^{j} \partial_{t}^{k} \psi_{b}\left(x, s_{l}+t\right) \rightarrow \partial_{x}^{j} \partial_{t}^{k} \gamma(x, t), \quad x \neq 0, \quad t \in \mathbb{R} .
$$

for some $\gamma \in C_{b}\left(\mathbb{R}, H^{1}\right)$. The convergence in (6.14) is uniform in $x$ and $t$ as long as $|x|+|t| \leq R$, for any $R>0$.

We call omega-limit trajectory any function $\gamma(x, t)$ that can appear as a limit in (6.14). Previous analysis demonstrates that the long-time asymptotics of the solution $\psi(x, t)$ in $H_{l o c}^{1}$ depends only on the bound component $\psi_{b}(x, t)$. By Corollary 6.2 , to conclude the proof of Theorem 2.9, it suffices to check that every omega-limit trajectory belongs to the set of solitary waves; that is,

$$
\begin{aligned}
\gamma(x, t)=C_{1} & \left(\begin{array}{c}
e^{-\varkappa_{1}^{+}|x|}+\frac{m e^{-\varkappa_{1}^{+}|x|}-\varkappa_{1} e^{-m|x|}}{\omega_{1}^{+}} \\
\varkappa_{1}^{+} \operatorname{sgn} x \frac{e^{-\varkappa_{1}^{+}|x|}-e^{-m|x|}}{\omega_{1}^{+}}
\end{array}\right) e^{-i \omega_{1}^{+} t} \\
& +C_{2}\left(\begin{array}{c}
-\varkappa_{2}^{+} \operatorname{sgn} x \frac{e^{-\varkappa_{2}^{+}|x|}-e^{-m|x|}}{\omega_{2}^{+}} \\
e^{-\varkappa_{2}^{+}|x|}-\frac{m e^{-\varkappa_{2}^{+}|x|}-\varkappa_{2}^{+} e^{-m|x|}}{\omega_{2}^{+}}
\end{array}\right) e^{-i \omega_{2}^{+} t}, \quad \varkappa_{j}^{+}=\sqrt{m^{2}-\left(\omega_{j}^{+}\right)^{2}}
\end{aligned}
$$

with some $\omega_{1}^{+}, \omega_{2}^{+} \in[-m, m]$. 
Spectral identity for omega-limit trajectories. Here we study the time spectrum of the omega-limit trajectories.

Definition 6.3. Let $\mu$ be a tempered distribution. By $\operatorname{Spec} \mu$ we denote the support of its Fourier transform:

$$
\operatorname{Spec} \mu:=\operatorname{supp} \tilde{\mu} \text {. }
$$

Proposition 6.4. 1. For any omega-limit trajectory $\gamma(x, t)$, the following spectral representation holds:

$$
\begin{aligned}
\gamma(x, t)= & \frac{1}{2 \pi}\left\langle\hat{p}(\omega) e^{-\varkappa(\omega)|x|}, e^{-i \omega t}\right\rangle \\
& +\frac{1}{2 \pi}\left\langle\hat{q}(x, \omega) \frac{e^{-\varkappa(\omega)|x|}-e^{-m|x|}}{2 \omega}, e^{-i \omega t}\right\rangle, \quad x \in \mathbb{R}, \quad t \in \mathbb{R},
\end{aligned}
$$

where $\hat{p}(\omega)$ and $\hat{q}(x, \omega)=(\beta-\alpha \operatorname{sgn} x) \sigma^{-1}(\omega) \hat{p}(\omega)$ are quasimeasures for all $x \in \mathbb{R}$, and

$$
\operatorname{supp} \hat{p} \subset[-m, m], \quad \operatorname{supp} \hat{q}(x) \subset[-m, m] .
$$

2. The following bound holds:

$$
\sup _{t \in \mathbb{R}}\|\gamma(\cdot, t)\|_{H^{1}}<\infty
$$

Note that, according to $(6.16), \hat{p}(\omega)$ is the Fourier transform of the function $p(t):=$ $\gamma(0, t), t \in \mathbb{R}$.

Proof. Formula (6.9) and representation (6.11) imply that

$$
\begin{aligned}
& \psi_{b}\left(x, s_{l}+t\right)=\frac{1}{2 \pi}\left\langle\hat{y}_{b}(\omega) e^{-\varkappa(\omega)|x|} e^{-i \omega s_{l}}, e^{-i \omega t}\right\rangle \\
& +\frac{1}{2 \pi}\left\langle\hat{h}_{b}(x, \omega) \frac{e^{-\varkappa(\omega)|x|}-e^{-m|x|}}{2 \omega} e^{-i \omega s_{l}}, e^{-i \omega t}\right\rangle, \quad x \neq 0, \quad t \in \mathbb{R} .
\end{aligned}
$$

Further, the convergence (6.14) and the bound (6.13) with $j=k=0$ imply that

$$
y_{b}\left(s_{l}+t\right) \rightarrow p(t), \quad s_{l} \rightarrow \infty,
$$

where $p \in C_{b}(\mathbb{R})$. The convergence is uniform on $[-T, T]$ for any $T>0$. Hence,

$$
\hat{y}_{b}(\omega) e^{-i \omega s_{l}} \rightarrow \hat{p}(\omega), \quad s_{l} \rightarrow \infty
$$

in the space of quasimeasures. Therefore,

$$
\hat{y}_{b}(\omega) e^{-\varkappa(\omega)|x|} e^{-i \omega s_{l}} \rightarrow \hat{p}(\omega) e^{-\varkappa(\omega)|x|}, \quad s_{l} \rightarrow \infty
$$

in the space of quasimeasures. Similarly,

$$
\begin{aligned}
& \hat{h}_{b}(x, \omega) \frac{e^{-\varkappa(\omega)|x|}-e^{-m|x|}}{2 \omega} e^{-i \omega s_{l}}=(\beta-\alpha \operatorname{sgn} x) \sigma^{-1}(\omega) \hat{y}_{b}(\omega) \frac{e^{-\varkappa(\omega)|x|}-e^{-m|x|}}{2 \omega} e^{-i \omega s_{l}} \\
& \rightarrow(\beta-\alpha \operatorname{sgn} x) \sigma^{-1}(\omega) \hat{p}(\omega) \frac{e^{-\varkappa(\omega)|x|}-e^{-m|x|}}{2 \omega}=\hat{q}(x, \omega) \frac{e^{-\varkappa(\omega)|x|}-e^{-m|x|}}{2 \omega}, \quad s_{l} \rightarrow \infty .
\end{aligned}
$$

Hence, the representation (6.16) follows from (6.19), (6.22) and (6.23); and (6.17) follows from (6.5). Finally, the bound (6.18) follows from (6.2) and (6.14). 
The relation (6.16) implies the basic spectral identity:

Corollary 6.5. For any omega-limit trajectory $\gamma(x, t)$,

$$
\operatorname{Spec} \gamma(x, \cdot)=\operatorname{Spec} p, \quad x \in \mathbb{R}
$$

\section{Nonlinear Spectral Analysis}

Here we will derive (6.15) from the following identity:

$$
p_{j}(t)=C_{j} e^{-i \omega_{j}^{+} t}, \quad j=1,2, \quad t \in \mathbb{R},
$$

which will be proved in three steps.

Step 1. The identity (7.1) is equivalent to $\hat{p}_{j}(\omega) \sim \delta\left(\omega-\omega_{j}^{+}\right)$, so we start with an investigation of Spec $p_{j}:=\operatorname{supp} \hat{p}_{j}$.

Lemma 7.1. For omega-limit trajectories the following spectral inclusion holds:

$$
\operatorname{Spec} F_{j}\left(p_{j}(\cdot)\right) \subset \operatorname{Spec} p_{j}, \quad j=1,2 .
$$

Proof. The convergence (6.14), Lemma 3.1 and Proposition 5.1 (ii) imply that the limiting trajectory $\gamma(x, t)$ is a solution to Eq. (1.1):

$$
i \dot{\gamma}(x, t)=D_{m} \gamma(x, t)-D_{m}^{-1} \delta(x) F(\gamma(0, t)), \quad(x, t) \in \mathbb{R}^{2} .
$$

Applying to both side operator $D_{m}$, we get

$$
i D_{m} \dot{\gamma}(x, t)=D_{m}^{2} \gamma(x, t)-\delta(x) F(\gamma(0, t)), \quad(x, t) \in \mathbb{R}^{2} .
$$

Since $\gamma(x, t)$ is smooth function for $x \leq 0$ and $x \geq 0$, we get the following algebraic identities :

$$
\gamma_{j}^{\prime}(0+, t)-\gamma_{j}^{\prime}(0-, t)=-F_{j}\left(p_{j}(t)\right), \quad t \in \mathbb{R}, \quad j=1,2 .
$$

The identities imply the spectral inclusion

$$
\operatorname{Spec} F_{j}\left(p_{j}(\cdot)\right) \subset \operatorname{Spec} \gamma_{j}^{\prime}(0+, \cdot) \cup \operatorname{Spec} \gamma_{j}^{\prime}(0-, \cdot)
$$

On the other hand, $\operatorname{Spec} \gamma_{j}^{\prime}(0+, \cdot) \cup \operatorname{Spec} \gamma_{k}^{\prime}(0-, \cdot) \subset \operatorname{Spec} p_{j}$ by (6.24). Therefore, (7.5) implies (7.2). 
Step 2.

Proposition 7.2. For any omega-limit trajectory, the following identity holds:

$$
\left|p_{j}(t)\right|=\text { const }, \quad j=1,2, \quad t \in \mathbb{R} .
$$

Proof. We are going to show that (7.6) follows from the key spectral relations (6.17), (7.2). Recall that the function $F_{j}(t):=F_{j}\left(p_{j}(t)\right)$ admits the representation [cf. (2.9)]

$$
F_{j}(t)=a_{j}(t) p_{j}(t), \quad j=1,2,
$$

where, according to (2.10),

$$
a_{j}(t)=-\sum_{n=1}^{N_{j}} 2 n_{j} u_{n, j}\left|p_{j}(t)\right|^{2 n-2}, \quad N_{j} \geq 2 ; \quad u_{N_{j}, j}>0 .
$$

Both functions $p_{j}(t)$ and $a_{j}(t)$ are bounded continuous functions in $\mathbb{R}$ by Proposition 6.4 (ii). Hence, $p_{j}(t)$ and $a_{j}(t)$ are tempered distributions. Furthermore, $\hat{p}_{j}$ and $\hat{\bar{p}}_{j}$ have the supports contained in $[-m, m]$ by (6.17). Hence, $a_{j}$ also has a bounded support since it is a sum of convolutions of finitely many $\hat{p}_{j}$ and $\hat{\bar{p}}_{j}$ by (7.8). Then the relation (7.7) translates into a convolution in the Fourier space, $\hat{F}_{j}=\hat{a}_{j} * \hat{p}_{j} /(2 \pi)$, and the spectral inclusion (7.2) takes the following form:

$$
\operatorname{supp} \hat{F}_{j}=\operatorname{supp} \hat{a}_{j} * \hat{p}_{j} \subset \operatorname{supp} \hat{p}_{j}
$$

Let us denote $\mathbf{F}_{j}=\operatorname{supp} \hat{F}_{j}, \mathbf{A}_{j}=\operatorname{supp} \hat{a}_{j}$, and $\mathbf{P}_{j}=\operatorname{supp} \hat{p}_{j}$. Then the spectral inclusion (7.9) reads as

$$
\mathbf{F}_{j} \subset \mathbf{P}_{j}
$$

On the other hand, it is well-known that $\operatorname{supp} \hat{a}_{j} * \hat{p}_{j} \subset \operatorname{supp} \hat{a}_{j}+\operatorname{supp} \hat{p}_{j}$, or $\mathbf{F}_{j} \subset$ $\mathbf{A}_{j}+\mathbf{P}_{j}$. Moreover, the Titchmarsh convolution theorem (see [12, Theorem 4.3.3]) imply that

$$
\inf \mathbf{F}_{j}=\inf \mathbf{A}_{j}+\inf \mathbf{P}_{j}, \quad \sup \mathbf{F}_{j}=\sup \mathbf{A}_{j}+\sup \mathbf{P}_{j}
$$

Now (7.10) and (7.11) result in

$$
\inf \mathbf{F}_{j}=\inf \mathbf{A}_{j}+\inf \mathbf{P}_{j} \geq \inf \mathbf{P}_{j}, \quad \sup \mathbf{F}_{j}=\sup \mathbf{A}_{j}+\sup \mathbf{P}_{j} \leq \sup \mathbf{P}_{j}
$$

so that inf $\mathbf{A}_{j} \geq 0 \geq \sup \mathbf{A}_{j}$. Thus, we conclude that supp $\hat{a}_{j}=\mathbf{A}_{j} \subset\{0\}$, therefore the distribution $\hat{a}_{j}(\omega)$ is a finite linear combination of $\delta(\omega)$ and its derivatives. Then $a_{j}(t)$ are polynomial in $t$; since $a_{j}(t)$ is bounded by Proposition 6.4 (ii), we conclude that $a_{j}(t)$ is constant. Now the relation (7.6) follows since $a_{j}(t)$ is a polynomial in $\left|p_{j}(t)\right|$, and its degree is strictly positive by (7.8). 
Step 3. Now the same Titchmarsh arguments imply that $P_{j}:=\operatorname{Spec} p_{j}$ is a point $\omega_{j}^{+} \in$ $[-m, m]$. Indeed, (7.6) means that $p_{j}(t) \bar{p}_{j}(t) \equiv C_{j}$, hence in the Fourier transform $\hat{p}_{j} * \hat{\bar{p}}_{j}=2 \pi C_{j} \delta(\omega)$. Therefore, if $p_{j}$ is not identically zero, the Titchmarsh Theorem implies that

$$
0=\sup P_{j}+\sup \left(-P_{j}\right)=\sup P_{j}-\inf P_{j}
$$

Hence $\inf P_{j}=\sup P_{j}$ and therefore $P_{j}=\omega_{k}^{+} \in[-m, m]$, so that $\hat{p}_{j}(\omega)$ is a finite linear combination of $\delta\left(\omega-\omega_{j}^{+}\right)$and its derivatives. As the matter of fact, the derivatives could not be present because of the boundedness of $p_{j}(t)=\gamma_{j}(0, t)$ that follows from Proposition 6.4 (ii). Thus, $\hat{p}_{j} \sim \delta\left(\omega-\omega_{j}^{+}\right)$, which implies (7.1).

Conclusion of the proof of Theorem 2.9 According to (7.1) and (6.23)

$$
\begin{aligned}
\hat{q}(\omega, x) & =(\beta-\alpha \operatorname{sgn} x) \sigma^{-1}(\omega) \hat{p}(\omega) \\
& =2 \pi(\beta-\alpha \operatorname{sgn} x)\left(\begin{array}{l}
C_{1}\left(\varkappa_{1}^{+}+m-\omega_{1}^{+}\right) \delta\left(\omega-\omega_{1}^{+}\right) \\
C_{2}\left(\varkappa_{2}^{+}+\omega_{2}^{+}+m\right) \delta\left(\omega-\omega_{2}^{+}\right)
\end{array}\right),
\end{aligned}
$$

where

$$
\varkappa_{j}^{+}=\sqrt{m^{2}-\left(\omega_{j}^{+}\right)^{2}}
$$

Then the representation (6.16) implies

$$
\left\{\begin{array}{l}
\gamma_{1}(x, t)=C_{1} e^{-i \omega_{1}^{+} t}\left(e^{-\varkappa_{1}^{+}|x|}+\left(\varkappa_{1}^{+}+m-\omega_{1}^{+}\right) \frac{e^{-\varkappa_{1}^{+}|x|}-e^{-m|x|}}{2 \omega_{1}^{+}}\right)-C_{2} e^{-i \omega_{2}^{+} t} \operatorname{sgn} x\left(\varkappa_{2}^{+}+m+\omega_{2}^{+}\right) \frac{e^{-\varkappa_{2}^{+}|x|}-e^{-m|x|}}{2 \omega_{2}^{+}} \\
\gamma_{2}(x, t)=C_{2} e^{-i \omega_{2}^{+} t}\left(e^{-\varkappa_{2}^{+}|x|}-\left(\varkappa_{2}^{+}+m+\omega_{2}^{+}\right) \frac{e^{-\varkappa_{2}^{+}|x|}-e^{-m|x|}}{2 \omega_{2}^{+}}\right)+C_{1} e^{-i \omega_{1}^{+} t} \operatorname{sgn} x\left(\varkappa_{1}^{+}+m-\omega_{1}^{+}\right) \frac{e^{-\varkappa_{1}^{+}|x|}-e^{-m|x|}}{2 \omega_{1}^{+}}
\end{array} \mid .\right.
$$

After simple evaluation, (7.13) becomes

$$
\left\{\begin{array}{l}
\gamma_{1}(x, t)=C_{1}^{\prime}\left(e^{-\varkappa_{1}^{+}|x|}+\frac{m e^{-\varkappa_{1}^{+}|x|}-\varkappa_{1} e^{-m|x|}}{\omega_{1}^{+}}\right) e^{-i \omega_{1}^{+} t}-C_{2}^{\prime} \varkappa_{2}^{+} \operatorname{sgn} x \frac{e^{-\varkappa_{2}^{+}|x|}-e^{-m|x|}}{\omega_{2}^{+}} e^{-i \omega_{2}^{+} t} \\
\gamma_{2}(x, t)=C_{1}^{\prime} \varkappa_{1}^{+} \operatorname{sgn} x \frac{e^{-\varkappa_{1}^{+}|x|}-e^{-m|x|}}{\omega_{1}^{+}} e^{-i \omega_{1}^{+} t}+C_{2}^{\prime}\left(e^{-\varkappa_{2}^{+}|x|}-\frac{m e^{-\varkappa_{2}^{+}|x|}-\varkappa_{2} e^{-m|x|}}{\omega_{2}^{+}}\right) e^{-i \omega_{2}^{+} t}
\end{array}\right.
$$

where we denote

$$
C_{1}^{\prime}=C_{1} \frac{\varkappa_{1}^{+}+m-\omega_{1}^{+}}{2 \varkappa_{1}^{+}}, \quad C_{2}^{\prime}=C_{2} \frac{\varkappa_{2}^{+}+m+\omega_{2}^{+}}{2 \varkappa_{2}^{+}} .
$$

Therefore, $\gamma(x, t)$ is a solitary wave (2.14). Due to Lemma 3.1 and Proposition 5.6 it remains to prove that

$$
\lim _{t \rightarrow \infty} \operatorname{dist}_{H_{l o c}^{1}}\left(\psi_{b}(t), \mathscr{S}\right)=0 \text {. }
$$

Assume by contradiction that there exists a sequence $s_{l} \rightarrow \infty$ such that

$$
\operatorname{dist}_{H_{l o c}^{1}}\left(\psi_{b}\left(s_{l}\right), \mathscr{S}\right) \geq \delta, \quad \forall l \in \mathbb{N}
$$


for some $\delta>0$. According to Corollary 6.2, there exist a subsequence $s_{l n}$ of the sequence $s_{l}, \omega_{1}^{+}, \omega_{2}^{+} \in \mathbb{R}$ and vector-function $\gamma(x, t)$, defined in (7.13) such that the following convergence hold

$$
\psi_{b}\left(x, t+s_{l_{n}}\right) \rightarrow \gamma(x, t), \quad l_{n} \rightarrow \infty, \quad t \in \mathbb{R} .
$$

This implies that

$$
\psi_{b}\left(x, s_{l_{n}}\right) \rightarrow \gamma(x, 0)=\phi_{\omega_{1}^{+}}(x)+\phi_{\omega_{2}^{+}}(x), \quad l_{n} \rightarrow \infty,
$$

where

$$
\begin{aligned}
& \phi_{\omega_{1}^{+}}(x)=C_{1}\left(\begin{array}{c}
e^{-\varkappa_{1}^{+}|x|}+\frac{m e^{-\varkappa_{1}^{+}|x|}-\varkappa_{1} e^{-m|x|}}{\omega_{1}^{+}} \\
\varkappa_{1}^{+} \operatorname{sgn} x \frac{e^{-\varkappa_{1}^{+}|x|}-e^{-m|x|}}{\omega_{1}^{+}}
\end{array}\right), \\
& \phi_{\omega_{2}^{+}}(x)=C_{2}\left(\begin{array}{c}
-\varkappa_{2}^{+} \operatorname{sgn} x \frac{e^{-\varkappa_{2}^{+}|x|}-e^{-m|x|}}{\omega_{2}^{+}} \\
e^{-\varkappa_{2}^{+}|x|}-\frac{m e^{-\varkappa_{2}^{+}|x|}-\varkappa_{2}^{+} e^{-m|x|}}{\omega_{2}^{+}}
\end{array}\right) .
\end{aligned}
$$

The convergence (7.16) contradicts to (7.15). This completes the proof of Theorem 2.9.

Acknowledgement. Open access funding provided by Austrian Science Fund (FWF).

Open Access This article is distributed under the terms of the Creative Commons Attribution 4.0 International License (http://creativecommons.org/licenses/by/4.0/), which permits unrestricted use, distribution, and reproduction in any medium, provided you give appropriate credit to the original author(s) and the source, provide a link to the Creative Commons license, and indicate if changes were made.

Publisher's Note Springer Nature remains neutral with regard to jurisdictional claims in published maps and institutional affiliations.

\section{A Global Well-Posedness}

Here we prove Theorem 2.1. We first need to adjust the nonlinearity $F$ so that it becomes bounded, together with its derivatives. Define

$$
\Lambda\left(\psi_{0}\right)=\sqrt{\frac{\mathscr{H}\left(\psi_{0}\right)-A}{m-B}},
$$

where $\psi_{0} \in H^{1}$ is the initial data from Theorem 2.1 and $A, B$ are constants from (2.4). Then we may pick a modified potential function $\widetilde{U} \in C^{2}\left(\mathbb{C}^{2}\right)$, so that

$$
\tilde{U}(\zeta)=U(\zeta) \quad \text { for }|\zeta| \leq \Lambda\left(\psi_{0}\right), \quad \zeta \in \mathbb{C}^{2},
$$

$\widetilde{U}(\zeta)$ satisfies (2.4) with the same constants $A, B$ as $U(\zeta)$ does:

$$
\tilde{U}(\zeta) \geq A-B|\zeta|^{2}, \quad \text { for } \zeta \in \mathbb{C}^{2}, \quad \text { where } A \in \mathbb{R} \text { and } 0 \leq B<m \text {, }
$$

and so that $|\widetilde{U}(\zeta)|,\left|\widetilde{U}^{\prime}(\zeta)\right|$, and $\left|\widetilde{U}^{\prime \prime}(\zeta)\right|$ are bounded for $\zeta \in \mathbb{C}^{2}$. We define

$$
\widetilde{F}_{j}(\zeta)=-\partial_{\bar{\zeta}_{j}} \tilde{U}(\zeta), \quad \zeta \in \mathbb{C}^{2},
$$


and consider the Cauchy problem of type (1.1) with the modified nonlinearity,

$$
\left\{\begin{array}{l}
i \dot{\psi}(x, t)=D_{m} \psi(x, t)-D_{m}^{-1} \delta(x) \widetilde{F}(\psi(0, t)), \quad x \in \mathbb{R}, \quad t \in \mathbb{R} . \\
\left.\psi\right|_{t=0}=\psi_{0}(x),
\end{array}\right.
$$

This is a Hamiltonian system, with the Hamilton functional

$$
\widetilde{\mathscr{H}}(\psi)=\frac{1}{2}\left\langle\psi,\left(-\partial_{x}^{2}+m^{2}\right) \psi\right\rangle+\widetilde{U}(\psi(0, t)), \quad \psi \in H^{1},
$$

which is Fréchet differentiable in the space $H^{1}$. By the Sobolev embedding theorem, $\|\psi\|_{L^{\infty}}^{2} \leq \frac{1}{2}\|\psi\|_{H^{1}}^{2}$. Moreover,

$$
\|\psi\|_{L^{\infty}}^{2} \leq \frac{1}{2 m}\|\psi\|^{2},
$$

where $\|\psi \mid\|^{2}:=\left\|\psi^{\prime}\right\|_{L^{2}}^{2}+m^{2}\|\psi\|_{L^{2}}^{2}$. Indeed, the Cauchy- Schwarz inequality and the Parseval identity imply

$$
\begin{aligned}
\|\psi\|_{L^{\infty}} & \leq \frac{1}{2 \pi}\|\tilde{\psi}\|_{L^{1}} \leq \frac{1}{2 \pi}\left(\int|\tilde{\psi}(k)|^{2}\left(m^{2}+k^{2}\right) d k\right)^{1 / 2}\left(\int \frac{d k}{m^{2}+k^{2}}\right)^{1 / 2} \\
& \leq \frac{1}{2 \pi} \sqrt{2 \pi}\|\psi\| \sqrt{\frac{\pi}{m}}=\frac{1}{\sqrt{2 m}}\|\psi\| .
\end{aligned}
$$

Thus (A.3) leads to

$$
\widetilde{U}(\psi(0)) \geq A-B\|\psi\|_{L^{\infty}}^{2} \geq A-\frac{B}{2 m}\|\psi\|^{2} .
$$

Taking into account (A.5), we obtain the inequality

$$
\|\psi\|^{2}=2 \widetilde{\mathscr{H}}(\psi)-2 \widetilde{U}(\psi(0)) \leq 2 \widetilde{\mathscr{H}}(\psi)-2 A+\frac{B}{m}\|\psi\|^{2}, \quad \psi \in H^{1},
$$

which implies

$$
\|\psi\|^{2} \leq \frac{2 m}{m-B}(\widetilde{\mathscr{H}}(\psi)-A), \quad \psi \in H^{1} .
$$

Lemma A.1. 1. $\widetilde{\mathscr{H}}\left(\psi_{0}\right)=\mathscr{H}\left(\psi_{0}\right)$.

2. If $\psi \in H^{1}$ satisfies $\mathscr{H}(\psi) \leq \mathscr{H}\left(\psi_{0}\right)$, then $\widetilde{U}(\psi(0))=U(\psi(0))$.

Proof. 1. According to (A.6), (A.7), and the choice of $\Lambda\left(\psi_{0}\right)$ in (A.1),

$$
\left\|\psi_{0}\right\|_{L^{\infty}}^{2} \leq \frac{1}{2 m}\left\|\psi_{0}\right\|^{2} \leq \frac{\mathscr{H}\left(\psi_{0}\right)-A}{m-B}=\Lambda^{2}\left(\psi_{0}\right)
$$

Thus, according to the choice of $\widetilde{U}$ (equality [A.2)], $\widetilde{U}\left(\psi_{0}(0)\right)=U\left(\psi_{0}(0)\right)$, proving (i).

2. By (A.6), (A.7), the condition $\widetilde{\mathscr{H}}(\psi) \leq \widetilde{\mathscr{H}}\left(\psi_{0}\right)$, and part $(i)$ of this lemma, we have:

$$
\|\psi\|_{L^{\infty}}^{2} \leq \frac{1}{2 m}\left\|\psi_{0}\right\|^{2} \leq \frac{\mathscr{H}(\psi)-A}{m-B} \leq \frac{\mathscr{H}\left(\psi_{0}\right)-A}{m-B}=\Lambda^{2}\left(\psi_{0}\right) .
$$

Hence, (ii) follows by (A.2).

Remark A.2. We will show that if $\psi(t)$ solves (A.4), then $\widetilde{\mathscr{H}}(\psi(t))=\widetilde{\mathscr{H}}\left(\psi_{0}\right)$, and therefore $\widetilde{U}(\psi(0, t))=U(\psi(0, t))$ by Lemma A.1 $(i i)$. Hence, $\widetilde{F}(\psi(0, t))=F(\psi(0, t))$ for all $t \geq 0$, allowing us to conclude that $\psi(t)$ solves (1.1) as well as (A.4). 
Local well-posedness. Denote by $e^{-i D_{m} t}$ the dynamical group of the free Dirac equation. Then the solution to the Cauchy problem (A.4) can be represented by

$$
\begin{aligned}
\psi(t) & =e^{-i D_{m} t} \psi_{0}+Z[\psi(0, \cdot)](t), \\
Z[\psi(0, \cdot)](t) & :=\int_{0}^{t} e^{-i D_{m}(t-s)} D_{m}^{-1} \delta(\cdot) \widetilde{F}(\psi(0, s)) d s, \quad t \in \mathbb{R} .
\end{aligned}
$$

The next lemma establishes the contraction principle for the integral equation (A.8).

Lemma A.3. There exists a constant $C>0$ so that for any two functions $\psi_{k}(\cdot, t) \in$ $C\left([-1,1], H^{1}\right), k=1,2$, one has:

$$
\begin{aligned}
& \left\|Z\left[\psi_{1}(0, \cdot)\right](t)-Z\left[\psi_{2}(0, \cdot)\right](t)\right\|_{H^{1}} \\
& \quad \leq C|t|^{1 / 2} \sup _{|s| \leq|t|}\left\|\psi_{1}(\cdot, s)-\psi_{2}(\cdot, s)\right\|_{H^{1}}, \quad|t| \leq 1 .
\end{aligned}
$$

Proof. It suffices to consider $t \geq 0$. In this case,

$$
e^{-i D_{m} t}=\left(i \partial_{t}+D_{m}\right) \mathscr{G}(t)
$$

where $\mathscr{G}(t)$ is the integral operator with the integral kernel

$$
\mathscr{G}(x, y, t)=G(x-y, t)=\theta(t-|x-y|) J_{0}\left(m \sqrt{t^{2}-(x-y)^{2}}\right) / 2 .
$$

Here $J_{0}$ is the Bessel function of zero order, and $\theta$ is the Heaviside function. According to (A.8) and (A.9),

$$
Z\left[\psi_{1}(0, \cdot)\right](t)-Z\left[\psi_{2}(0, \cdot)\right](t)=I_{1}(x, t)+I_{2}(x, t),
$$

where

$$
\begin{aligned}
& I_{1}(x, t):=\int_{0}^{t} G(x, t-s)\left(\widetilde{F}\left(\psi_{1}(0, s)\right)-\widetilde{F}\left(\psi_{2}(0, s)\right)\right) d s \\
& I_{2}(x, t):=i \int_{0}^{t}\left[\dot{G}(\cdot, t-s) * D_{m}^{-1} \delta(\cdot)\right](x)\left(\widetilde{F}\left(\psi_{1}(0, s)\right)-\widetilde{F}\left(\psi_{2}(0, s)\right) d s .\right.
\end{aligned}
$$

First we prove the $L_{2}$ estimate for $I_{j}(x, t)$. By the Sobolev embedding theorem,

$$
\begin{aligned}
\left\|I_{1}(\cdot, t)\right\|_{L^{2}} & \leq \sup _{s \in[0, t]}\left|\widetilde{F}\left(\psi_{1}(0, s)\right)-\widetilde{F}\left(\psi_{2}(0, s)\right)\right| \int_{0}^{t}\|G(\cdot, s)\|_{L^{2}} d s \\
& \leq C \sup _{z \in \mathbb{C}}|\nabla \widetilde{F}(z)|\left\|\psi_{1}(\cdot, s)-\psi_{2}(\cdot, s)\right\|_{H^{1}} \int_{0}^{t}\left\|\frac{\sin s \sqrt{\xi^{2}+m^{2}}}{\sqrt{\xi^{2}+m^{2}}}\right\|_{L^{2}} d s \\
& \leq C_{1} t \sup _{s \in[0, t]}\left\|\psi_{1}(\cdot, s)-\psi_{2}(\cdot, s)\right\|_{H^{1}},
\end{aligned}
$$

where we took into account that $|\nabla \widetilde{F}(z)|$ is bounded due to the choice of $\widetilde{U}$. Similarly,

$$
\begin{aligned}
\left\|I_{2}(\cdot, t)\right\|_{L^{2}} & \leq C \sup _{s \in[0, t]}\left\|\psi_{1}(\cdot, s)-\psi_{2}(\cdot, s)\right\|_{H^{1}} \int_{0}^{t}\left\|(-i \xi \alpha+m \beta) \frac{\cos s \sqrt{\xi^{2}+m^{2}}}{\xi^{2}+m^{2}}\right\|_{L^{2}} d s \\
& \leq C_{1} t \sup _{s \in[0, t]}\left\|\psi_{1}(\cdot, s)-\psi_{2}(\cdot, s)\right\|_{H^{1}} .
\end{aligned}
$$


Now, we derive the $L^{2}$ estimates for the derivatives $\partial_{x} I_{1}(x, t)$ and $\partial_{x} I_{2}(x, t)$. We have

$$
\partial_{x} G(x, t)=\frac{1}{2} \theta(t-|x|) \partial_{x} J_{0}\left(m \sqrt{t^{2}-x^{2}}\right)-\frac{1}{2} \delta(t-|x|) \operatorname{sgn} x
$$

where

$$
\left|\partial_{x} J_{0}\left(m \sqrt{t^{2}-x^{2}}\right)\right|=\left|J_{1}\left(m \sqrt{t^{2}-x^{2}}\right) \frac{m x}{\sqrt{t^{2}-x^{2}}}\right| \leq C, \quad|x| \leq|t| \leq 1 .
$$

Hence,

$$
\begin{aligned}
\left\|\partial_{x} I_{1}(\cdot, t)\right\|_{L^{2}} & \leq C_{1}\left\|\int_{0}^{t}(C \theta(t-s-|x|)+\delta(t-s-|x|)) d s\right\|_{L^{2}} \sup _{s \in[0, t]}\left\|\psi_{1}(\cdot, s)-\psi_{2}(\cdot, s)\right\|_{H^{1}} \\
& \leq C_{2}\left[t\|\theta(t-|x|)\|_{L^{2}}+\|\theta(t-|x|)\|_{L^{2}}\right] \sup _{s \in[0, t]}\left\|\psi_{1}(\cdot, s)-\psi_{2}(\cdot, s)\right\|_{H^{1}} \\
& \leq C_{2} t^{1 / 2}(t+1) \sup _{s \in[0, t]}\left\|\psi_{1}(\cdot, s)-\psi_{2}(\cdot, s)\right\|_{H^{1}} .
\end{aligned}
$$

Further,

$$
\begin{aligned}
\partial_{x} D_{m}^{-1} \delta(x) & =\partial_{x} D_{m} D_{m}^{-2} \delta(x)=\frac{1}{2 \pi} \int_{\mathbb{R}} \frac{e^{-i \xi x}(-i \xi)(-i \xi \alpha+m \beta) d \xi}{\xi^{2}+m^{2}} \\
& =-\alpha \delta(x)+m^{2} \alpha D_{m}^{-2} \delta(x)+m \beta \partial_{x} D_{m}^{-2} \delta(x)
\end{aligned}
$$

Hence,

$$
\begin{aligned}
\partial_{x} I_{2}(x, t)= & -i \alpha \int_{0}^{t} \dot{G}(x, t-s)\left(\widetilde{F}\left(\psi_{1}(0, s)\right)-\widetilde{F}\left(\psi_{2}(0, s)\right)\right) d s \\
& +i m^{2} \alpha \int_{0}^{t}\left[\dot{G}(\cdot, t-s) * D_{m}^{-2} \delta(\cdot)\right](x) \alpha\left(\widetilde{F}\left(\psi_{1}(0, s)\right)-\widetilde{F}\left(\psi_{2}(0, s)\right) d s\right. \\
& +i m \beta \int_{0}^{t} \partial_{x}\left[\dot{G}(\cdot, t-s) * D_{m}^{-2} \delta(\cdot)\right](x)\left(\widetilde{F}\left(\psi_{1}(0, s)\right)-\widetilde{F}\left(\psi_{2}(0, s)\right) d s\right. \\
= & \alpha J_{1}(x, t)+\alpha J_{2}(x, t)+\beta J_{3}(x, t) .
\end{aligned}
$$

The $L^{2}$ norm of $J_{1}(x, t)$ is estimated similarly to the $L^{2}$ norm of $\partial_{x} I_{1}(x, t)$. Further, similarly to (A.10), we get

$$
\begin{aligned}
\left\|J_{2}(\cdot, t)\right\|_{L^{2}} & \leq C \sup _{s \in[0, t]}\left\|\psi_{1}(\cdot, s)-\psi_{2}(\cdot, s)\right\|_{H^{1}} \int_{0}^{t}\left\|\frac{\cos s \sqrt{\xi^{2}+m^{2}}}{\xi^{2}+m^{2}}\right\|_{L^{2}} d s \\
& \leq C_{1} t \sup _{s \in[0, t]}\left\|\psi_{1}(\cdot, s)-\psi_{2}(\cdot, s)\right\|_{H^{1}} \\
\left\|J_{3}(\cdot, t)\right\|_{L^{2}} & \leq C \sup _{s \in[0, t]}\left\|\psi_{1}(\cdot, s)-\psi_{2}(\cdot, s)\right\|_{H^{1}} \int_{0}^{t}\left\|\frac{\xi \cos s \sqrt{\xi^{2}+m^{2}}}{\xi^{2}+m^{2}}\right\|_{L^{2}} d s \\
& \leq C_{1} t \sup _{s \in[0, t]}\left\|\psi_{1}(\cdot, s)-\psi_{2}(\cdot, s)\right\|_{H^{1}} .
\end{aligned}
$$

For $E>0$, let us denote $H_{E}^{1}=\left\{\psi_{0} \in H^{1}: \mathscr{H}\left(\psi_{0}\right) \leq E\right\}$.

Corollary A.4. 1. For any $E>0$ there exists $\tau=\tau(E)>0$ such that for any $\psi_{0} \in H_{E}^{1}$ there is a unique solution $\psi(x, t) \in C\left([-\tau, \tau], H^{1}\right)$ to the Cauchy problem (A.4) with the initial condition $\psi(0)=\psi_{0}$.

2. The maps $W(t): \psi_{0} \mapsto \psi(t), t \in[-\tau, \tau]$ are continuous from $H_{E}^{1}$ to $H^{1}$. 
Energy conservation and global well posedness.

Lemma A.5. For the solution to the Cauchy problem (A.4) with the initial data $\psi_{0} \in H^{1}$, the energy is conserved: $\widetilde{\mathscr{H}}(\psi(t))=$ const, $t \in[-\tau, \tau]$.

Proof. The Galerkin approximations provide a solution $\psi \in L^{\infty}\left(\mathbb{R}, H^{1}\right)$ to (A.4) with energy estimate

$$
\widetilde{\mathscr{H}}(\psi(t)) \leq \widetilde{\mathscr{H}}\left(\psi_{0}\right), \quad t \in \mathbb{R} .
$$

Moreover, estimates from the proof of Lemma A.3 imply that $\psi \in C\left(\mathbb{R}, H^{1}\right)$. Therefore,

$$
\mathscr{H}(\psi(t))=\mathscr{H}\left(\psi_{0}\right), \quad t \in[-\tau, \tau]
$$

since the inequality (A.14) also holds with $\psi(s)$ instead of $\psi_{0}$ for every $s \in[-\tau, \tau]$ by the uniqueness of solutions proved in Corollary A.4.

Corollary A.6. 1. The solution $\psi$ to the Cauchy problem (A.4) with the initial data $\left.\psi\right|_{t=0}=\psi_{0} \in H^{1}$ exists globally: $\psi \in \underset{C_{b}}{C_{t}}\left(\mathbb{R}, H^{1}\right)$.

2. The energy is conserved: $\widetilde{\mathscr{H}}(\psi(t))=\widetilde{\mathscr{H}}\left(\psi_{0}\right), \quad t \in \mathbb{R}$.

Proof. Corollary A.4 (i) yields a solution $\psi \in C\left([-\tau, \tau], H^{1}\right)$ with a positive $\tau=\tau(E)$. However, the value of $\mathscr{H}(\psi(t))$ is conserved for $t \leq \tau$ by Lemma A.5. Corollary A.4 $(i)$ allows then to extend $\psi$ to the interval $[-2 \tau, 2 \tau]$, and eventually to all $t \in \mathbb{R}$.

Conclusion of the proof of Theorem 2.1. The trajectory $\psi \in C_{b}\left(\mathbb{R}, H^{1}\right)$ is a solution to (A.4), for which Corollary A.6 (ii) together with Lemma A.1 (i) imply the energy conservation (2.5). By Lemma A.1 (ii), $U(\psi(0, t))=U(\psi(0, t))$, for all $t \in \mathbb{R}$. This tells us that $\psi(x, t)$ is a solution to (1.1). Finally, the a priori bound (2.6) follows from (A.7) and the conservation of $\mathscr{H}(\psi(t))$. This finishes the proof of Theorem 2.1.

\section{B Free Dirac Equation}

Here we show that the function

$$
\begin{aligned}
\varphi^{ \pm}(x, t) & =\frac{1}{2 \pi} \int_{\mathbb{R}}\left(I+\frac{m}{\omega(k)} \beta \pm \frac{i k}{\omega(k)} \alpha\right) \frac{\hat{f}_{d}(\omega(k))}{2 i k} e^{ \pm i k x} e^{-i \omega(k) t} \frac{k d k}{\omega(k)}, \\
\omega(k) & =\sqrt{k^{2}+m^{2}}
\end{aligned}
$$

are the solutions to the free Dirac equation (3.1). It suffices to prove that for any $q=$ $\left(q_{1}, q_{2}\right) \in \mathbb{C}^{2}$ the functions

$$
p^{ \pm}(x, t)=e^{ \pm i k x} e^{-i \omega(k) t}(\omega(k)+m \beta \pm i k \alpha) q
$$

satisfy

$$
\left(i \partial_{t}-D_{m}\right) p^{ \pm}(x, t)=0 .
$$

Indeed, the functions $u_{j}^{ \pm}(x, t)=e^{ \pm i k x} e^{-i \omega(k) t} q_{j}$ obviously satisfy

$$
\ddot{u}_{j}^{ \pm}(x, t)+D_{m}^{2} u_{j}^{ \pm}(x, t)=0 .
$$


Moreover,

$$
\begin{aligned}
p^{ \pm}(x, t)= & (\omega(k) I+m \beta \pm i k \alpha) u^{ \pm}(x, t)=\left(i \partial_{t}+D_{m}\right) u^{ \pm}(x, t), \\
& u^{ \pm}(x, t)=\left(u_{1}^{ \pm}(x, t), u_{2}^{ \pm}(x, t)\right) .
\end{aligned}
$$

Hence,

$$
\left(i \partial_{t}-D_{m}\right) p^{ \pm}(x, t)=\left(i \partial_{t}-D_{m}\right)\left(i \partial_{t}+D_{m}\right) u^{ \pm}(x, t)=-\ddot{u}^{ \pm}(x, t)-D_{m}^{2} u^{ \pm}(x, t)=0 .
$$

\section{Proof of the Lemma 2.7}

It suffices to consider the case $j=1$ only, since in the Eq. (2.13) with $j=1$ and $j=2$ are similar. For $\omega_{1} \in(-m, m)$ denote $\mu_{1}=\mu_{1}\left(\omega_{1}\right)=1+\frac{m-\sqrt{m^{2}-\omega_{1}^{2}}}{\omega_{1}}>0$. Then Eq. (2.13) for $j=1$ reads

$$
2 \varkappa_{1} C_{1}=F_{1}\left(\mu_{1} C_{1}\right), \quad \varkappa_{1}=\varkappa_{1}\left(\omega_{1}\right)=\sqrt{m^{2}-\omega_{1}^{2}}>0, \quad \omega_{1} \in(-m, m) .
$$

Taking into account (2.9)-(2.10), we rewrite this equation as

$$
2 \varkappa_{1}=a_{1}\left(\mu_{1}^{2}\left|C_{1}\right|^{2}\right) \mu_{1}
$$

where

$$
a_{1}\left(|\zeta|^{2}\right)=M-\sum_{n=2}^{N_{1}} 2 n u_{n, 1}|\zeta|^{2 n-2}, \quad N_{1} \geq 2, \quad M>0, \quad u_{N_{1}, 1}>0
$$

Equation (C.1) has nonzero solutions $C_{1}=C_{1}\left(\omega_{1}\right)$ in the case when $2 \varkappa_{1}\left(\omega_{1}\right)<$ $M \mu_{1}\left(\omega_{1}\right)$, i.e.

$$
2 \sqrt{m^{2}-\omega_{1}^{2}}<M\left(1+\frac{m-\sqrt{m^{2}-\omega_{1}^{2}}}{\omega_{1}}\right)
$$

Dividing by $\sqrt{m+\omega}>0$, we arrive at the inequality

$$
2 \sqrt{m-\omega_{1}}<M \frac{\sqrt{m+\omega_{1}}-\sqrt{m-\omega_{1}}}{\omega_{1}} .
$$

Obviously, (C.2) holds for any $M>0$ and $\omega_{1}$ sufficiently close to $m$. It is not difficult to verify that this holds for any $\omega_{1} \in(-m, m)$ in the case when $M>(1+\sqrt{2}) m$. Indeed, (C.2) is equivalent to

$$
\sqrt{m-\omega_{1}}<\frac{M}{\sqrt{m+\omega_{1}}+\sqrt{m-\omega_{1}}}
$$

or

$$
\sqrt{m^{2}-\omega_{1}^{2}}<M+\omega_{1}-m
$$

For $M>2 m$, squaring both sides, we obtain

$$
\begin{aligned}
& 2 \omega_{1}^{2}+2 \omega_{1}(M-m)+M^{2}-2 M m \\
& =2\left(\omega_{1}+\frac{M-m}{2}\right)^{2}+\frac{1}{2}(M-m(1+\sqrt{2}))(M+m(\sqrt{2}-1))>0,
\end{aligned}
$$

which holds for any $M>(1+\sqrt{2}) m$. 


\section{Linear Case}

Here we consider the linear case, when

$$
F_{j}\left(\zeta_{j}\right)=a_{j} \zeta_{j}, \quad U_{j}\left(\zeta_{j}\right)=-\frac{a_{j}}{2}\left|\zeta_{1}\right|^{2} \quad a_{j} \in \mathbb{R}
$$

Now Eq. (1.1) reads

$$
\dot{\psi}(x, t)=D_{m} \psi(x, t)-D_{m}^{-1} A \psi(0, t) \delta(x), \quad A=\left(\begin{array}{cc}
a_{1} & 0 \\
0 & a_{2}
\end{array}\right), \quad x \in \mathbb{R}, \quad t \in \mathbb{R} .
$$

We restrict our consideration to the case when $a_{j}<2 m, j=1,2$. It is in this case that condition (2.4) is satisfied, and then all conclusions of Theorem 2.1 on global wellposedness for Eq. (D.2) hold .

Let us calculate corresponding solitary waves. Now Eq. (2.13) become

$$
\begin{aligned}
& \sqrt{m^{2}-\omega_{1}^{2}}=a_{1} \frac{\omega_{1}+m-\sqrt{m^{2}-\omega_{1}^{2}}}{2 \omega_{1}}, \\
& \sqrt{m^{2}-\omega_{2}^{2}}=a_{2} \frac{\omega_{2}-m+\sqrt{m^{2}-\omega_{2}^{2}}}{2 \omega_{2}}, \quad \omega_{j} \in(-m, m) .
\end{aligned}
$$

Cancelling the nonzero factors $\sqrt{m+\omega_{1}}$ and $\sqrt{m-\omega_{2}}$, we obtain

$$
\sqrt{m-\omega_{1}}=a_{1} \frac{\sqrt{m+\omega_{1}}-\sqrt{m-\omega_{1}}}{2 \omega_{1}}, \quad \sqrt{m+\omega_{2}}=a_{2} \frac{\sqrt{m+\omega_{2}}-\sqrt{m-\omega_{2}}}{2 \omega_{2}} .
$$

Multiplying both sides of this equations by $\sqrt{m+\omega_{1}}+\sqrt{m-\omega_{1}}$ and $\sqrt{m+\omega_{2}}+$ $\sqrt{m-\omega_{2}}$, respectively, we get

$$
\sqrt{m^{2}-\omega_{1}^{2}}+m-\omega_{1}=a_{1}, \quad \sqrt{m^{2}-\omega_{2}^{2}}+m+\omega_{2}=a_{2}, \quad \omega_{j} \in(-m, m),
$$

where the left hand sides of both equalities are positive for $\omega_{j} \in(-m, m)$. Hence, there are no nonzero solitary waves for $a_{j} \leq 0, j=1,2$. For $0<a_{j}<2 m$, the corresponding equation of (D.3) has the unique solution

$$
\omega_{j}=\frac{(-1)^{j}}{2}\left(a_{j}-m-\sqrt{m^{2}-a_{j}^{2}+2 m a_{j}}\right) .
$$

Finally, we conclude that for $0<a_{j}<2 m, j=1,2$, the set of finite energy solitary waves is given by

$$
\mathscr{S}=\left\{C_{1}\left[\begin{array}{c}
e^{-\varkappa_{1}|x|}+\frac{m e^{-\varkappa_{1}|x|}-\varkappa_{1} e^{-m|x|}}{\omega_{1}} \\
\varkappa_{1} \operatorname{sgn} x \frac{e^{-\varkappa_{1}|x|}-e^{-m|x|}}{\omega_{1}}
\end{array}\right]+C_{2}\left[\begin{array}{c}
-\varkappa_{2} \operatorname{sgn} x \frac{e^{-\varkappa_{2}|x|}-e^{-m|x|}}{\omega_{2}} \\
e^{-\varkappa_{2}|x|}-\frac{m e^{-\varkappa_{2}|x|}-\varkappa_{2} e^{-m|x|}}{\omega_{2}}
\end{array}\right]: C_{1}, C_{2} \in \mathbb{C}\right\} .
$$

In the case $a_{1}<0$ the set $\mathscr{S}$ is given by (D.5) with $C_{1}=0$, while $C_{2} \in \mathbb{C}$ is arbitrary, and vice versa. . 
Theorem D.1. Assume that $F_{j}\left(\psi_{j}\right)=a_{j} \psi_{j}$, where $a_{j}<2 m, j=1,2$. Then for any $\psi_{0} \in H^{1}$ the solution $\psi(t) \in C\left(\mathbb{R}, H^{1}\right)$ to the Cauchy problem $(1.1)$ with $\psi(0)=\psi_{0}$ converges to $\mathscr{S}$ in the space $H_{\text {loc }}^{1}(\mathbb{R}) \otimes C^{2}$ :

$$
\Psi(t) \rightarrow \mathscr{S}, \quad t \rightarrow \pm \infty .
$$

Proof. We proceed as in the proof of Theorem 2.9 until we get Eq. (7.3), which takes now the following form:

$$
i \dot{\gamma}(x, t)=D_{m} \gamma(x, t)-D_{m}^{-1} A \gamma(0, t) \delta(x), \quad(x, t) \in \mathbb{R}^{2} .
$$

In this case we cannot use the Titchmarsh arguments since the condition (2.8) fails. Now we should prove that

$$
\gamma(\cdot, t) \in \mathscr{S}, \quad t \in \mathbb{R}
$$

for all solutions of (D.7) with the structure (6.16). In the Fourier transform $\hat{\gamma}(x, \omega)=$ $\mathscr{F}_{t \rightarrow \omega}[\gamma(x, t)]$ the Eq. (D.7) becomes

$$
\left(D_{m}-\omega\right) \hat{\gamma}(x, \omega)-D_{m}^{-1} A \hat{\gamma}(0, \omega) \delta(x)=0, \quad(x, \omega) \in \mathbb{R}^{2} .
$$

Applying the operator $D_{m}+\omega$, we get

$$
\left(D_{m}^{2}-\omega^{2}\right) \hat{\gamma}(x, \omega)-A \hat{\gamma}(0, \omega) \delta(x)-\omega D_{m}^{-1} A \hat{\gamma}(0, \omega) \delta(x)=0, \quad(x, \omega) \in \mathbb{R}^{2} .
$$

On the other hand, the representation (6.16) implies that

$$
\begin{aligned}
& \hat{\gamma}(x, \omega)=\hat{p}(\omega) e^{-\varkappa(\omega)|x|}+(\beta-\alpha \operatorname{sgn} x) \sigma^{-1}(\omega) \hat{p}(\omega) \frac{e^{-\varkappa(\omega)|x|}-e^{-m|x|}}{2 \omega}, \\
& \hat{\gamma}(0, \omega)=\hat{p}(\omega) .
\end{aligned}
$$

Substituting this into (D.9) and equating coefficients with delta functions, we obtain

$$
2 \varkappa(\omega) \hat{p}(\omega)+\beta \sigma^{-1}(\omega) \hat{p}(\omega) \frac{\varkappa(\omega)-m}{\omega}=A \hat{p}(\omega), \quad \varkappa=\sqrt{m^{2}-\omega^{2}},
$$

where $\sigma^{-1}(\omega)$ is the diagonal matrix (6.8) with matrix elements $\left[\sigma^{-1}(\omega)\right]_{j j}:=$ $v_{j}(\omega)=\varkappa(\omega)+m-(-1)^{j} \omega$.

Therefore, on the support of the distribution $\hat{p}_{j}(\omega), j=1,2$, the identity hold

$$
2 \varkappa(\omega)+(-1)^{j+1} v_{j}(\omega) \frac{\varkappa(\omega)-m}{\omega}=a_{j} .
$$

Simplifying, we arrive at the following equation

$$
\varkappa(\omega)+m+(-1)^{j} \omega=a_{j}
$$

which are exactly Eq. (D.3) for soliton parameters $\omega_{j}$. Finally, we obtain that for $0<$ $a_{j}<2 m$

$$
\operatorname{supp} \hat{p}_{j}=\omega_{j} \in(-m, m),
$$

where $\omega_{j}$ are given in (D.4). Hence, the inclusion (D.8) follows. This finishes the proof of Theorem D.1. 


\section{References}

1. Adami, R., Noja, D., Ortoleva, C.: Orbital and asymptotic stability for standing waves of a nonlinear Schrödinger equation with concentrated nonlinearity in dimension three. J. Math. Phys. 54(1), 013501 (2013)

2. Albeverio, S., Figari, R.: Quantum fields and point interactions. Rend. Mat. Appl. (7) 39, 161-180 (2018)

3. Berezin, F.A., Faddeev, L.D.: Remark on the Schrödinger equation with singular potential. Sov. Math. Dokl. 2, 372-375 (1961)

4. Buslaev, V., Perelman, G.: On the stability of solitary waves for nonlinear Schrödinger equations. In: Nonlinear Evolution Equations, Volume 164 of American Mathematical Society Translation 2, pp. 75-98. American Mathematical Society, Providence (1995)

5. Buslaev, V., Sulem, C.: On asymptotic stability of solitary waves for nonlinear Schrödinger equations. Ann. Inst. H. Poincaré Anal. Non Linéaire 20, 419-475 (2003)

6. Buslaev, V., Komech, A., Kopylova, E., Stuart, D.: On asymptotic stability of solitary waves in nonlinear Schrödinger equation. Commun. Partial Differ. Equ. 33(4), 669-705 (2008)

7. Cornish, F.H.: Classical radiation theory and point charges. Proc. Phys. Soc. 86(3), 427-442 (1965)

8. Cuccagna, S.: Stabilization of solutions to nonlinear Schrödinger equations. Commun. Pure Appl. Math. 54, 1110-1145 (2001)

9. Dirac, P.A.M.: Classical theory of radiating electrons. Proc. R. Soc. A 167, 148-169 (1938)

10. Erdelyi, A., et al.: Tables of Integral Transforms, vol. 1. McGraw-Hill Book Company, New York (1954)

11. Gittel, H.-P., Kijowski, J., Zeidler, E.: The relativistic dynamics of the combined particle-field system in renormalized classical electrodynamics. Commun. Math. Phys. 198, 711-736 (1998)

12. Hörmander, L.: The Analysis of Linear Partial Differential Operators. I. Springer Study Edition, 2nd edn. Springer, Berlin (1990)

13. Hörmander, L.: On the fully nonlinear Cauchy problem with small data. II. In: Microlocal Analysis and Nonlinear Waves (Minneapolis, MN, 1988-1989), Volume 30 of IMA Volumes in Mathematics and its Applications, pp. 51-81. Springer, New York (1991)

14. Klainerman, S.: Long-time behavior of solutions to nonlinear evolution equations. Arch. Ration. Mech. Anal. 78, 73-98 (1982)

15. Komech, A.: On stabilization of string-nonlinear oscillator interaction. J. Math. Anal. Appl. 196, 384-409 (1995)

16. Komech, A.: On transitions to stationary states in one-dimensional nonlinear wave equations. Arch. Ration. Mech. Anal. 149, 213-228 (1999)

17. Komech, A.I., Komech, A.A.: Global attractor for a nonlinear oscillator coupled to the Klein-Gordon field. Arch. Ration. Mech. Anal. 185, 105-142 (2007)

18. Komech, A.I., Komech, A.A.: Global attraction to solitary waves for Klein-Gordon equation with mean field interaction. Ann. l'IHP-ANL 26(3), 855-868 (2009)

19. Komech, A.I., Komech, A.A.: Global attraction to solitary waves for nonlinear Dirac equation with mean field interaction. SIAM J. Math. Anal. 42(6), 2944-2964 (2010)

20. Komech, A.I., Komech, A.A.: On global attraction to solitary waves for the Klein-Gordon field coupled to several nonlinear oscillators. J. Math. Pures Appl. 93, 91-111 (2010)

21. Komech, A., Kopylova, E., Stuart, D.: On asymptotic stability of solitons in a nonlinear Schrödinger equation. Commun. Pure Appl. Anal. 11(3), 1063-1079 (2012)

22. Komech, A., Spohn, H.: Long-time asymptotics for the coupled Maxwell-Lorentz equations. Commun. Partial Differ. Equ. 25, 559-584 (2000)

23. Komech, A., Spohn, H., Kunze, M.: Long-time asymptotics for a classical particle interacting with a scalar wave field. Commun. Partial Differ. Equ. 22, 307-335 (1997)

24. Komech, A., Vainberg, B.: On asymptotic stability of stationary solutions to nonlinear wave and KleinGordon equations. Arch. Ration. Mech. Anal. 134, 227-248 (1996)

25. Kopylova, E.: On global attraction to stationary state for wave equation with concentrated nonlinearity. J. Dyn. Differ. Equ. 30(1), 107-116 (2018)

26. Kopylova, E.: Global attraction to solitary waves for Klein-Gordon equation with concentrated nonlinearity. Nonlinearity 30(11), 4191-4207 (2017)

27. Morawetz, C., Strauss, W.: Decay and scattering of solutions of a nonlinear relativistic wave equation. Commun. Pure Appl. Math. 25, 1-31 (1972)

28. Soffer, A., Weinstein, M.: Multichannel nonlinear scattering for nonintegrable equations. Commun. Math. Phys. 133, 119-146 (1990)

29. Soffer, A., Weinstein, M.: Multichannel nonlinear scattering for nonintegrable equations. II. J. Differ. Equ. 98, 376-390 (1992). The case of anisotropic potentials and data

30. Soffer, A., Weinstein, M.: Resonances, radiation damping and instability in Hamiltonian nonlinear wave equations. Invent. Math. 136, 9-74 (1999) 
31. Spohn, H.: Dynamics of Charged Particles and their Radiation Field. Cambridge University Press, Cambridge (2004)

32. Strauss, W.: Decay and asymptotics for $\square u=f(u)$. J. Funct. Anal. 2, 409-457 (1968)

33. Yafaev, D.R.: On a zero-range interaction of a quantum particle with the vacuum. J. Phys. A 25, 963-978 (1992)

34. Yafaev, D.R.: A point interaction for the discrete Schrödinger operator and generalized Chebyshev polynomial. J. Math. Phys. 58(6), 063511 (2017)

35. Zel'dovich, Ya B.: Scattering by a singular potential in perturbation theory and in the momentum representation. JETP 11(3), 594-597 (1960)

Communicated by W. Schlag 